\title{
Elliptic polylogarithms and iterated integrals on elliptic curves. II. An application to the sunrise integral
}

\author{
Johannes Broedel, ${ }^{1, *}$ Claude Duhr, ${ }^{2,3,}$ Falko Dulat, ${ }^{4, \$}$ and Lorenzo Tancredi ${ }^{2, \S}$ \\ ${ }^{1}$ Institut für Mathematik und Institut für Physik, Humboldt-Universität zu Berlin, \\ IRIS Adlershof, Zum Großen Windkanal 6, 12489 Berlin, Germany \\ ${ }^{2}$ Theoretical Physics Department, CERN, CH-1211 Geneva 23, Switzerland \\ ${ }^{3}$ Center for Cosmology, Particle Physics and Phenomenology (CP3), Université catholique de Louvain, \\ Chemin du Cyclotron 2, 1348 Louvain-La-Neuve, Belgium \\ ${ }^{4}$ SLAC National Accelerator Laboratory, Stanford University, Stanford, California 94309, USA
}

(Received 9 January 2018; published 12 June 2018)

\begin{abstract}
We introduce a class of iterated integrals that generalize multiple polylogarithms to elliptic curves. These elliptic multiple polylogarithms are closely related to similar functions defined in pure mathematics and string theory. We then focus on the equal-mass and non-equal-mass sunrise integrals, and we develop a formalism that enables us to compute these Feynman integrals in terms of our iterated integrals on elliptic curves. The key idea is to use integration-by-parts identities to identify a set of integral kernels, whose precise form is determined by the branch points of the integral in question. These kernels allow us to express all iterated integrals on an elliptic curve in terms of them. The flexibility of our approach leads us to expect that it will be applicable to a large variety of integrals in high-energy physics.
\end{abstract}

DOI: 10.1103/PhysRevD.97.116009

\section{INTRODUCTION}

With the discovery of the Higgs boson at the Large Hadron Collider (LHC) at CERN and the absence of signals of physics beyond the Standard Model (SM), we have entered a new era of precision physics. Indeed, the mass of the Higgs boson was the last free parameter of the SM whose value had not yet been determined by experiments. Therefore, with the measurement of the Higgs mass, the SM is a fully predictive theory that can be valid, at least in principle, all the way up to the Planck scale. Even if the scale of new physics is beyond the reach of the LHC, small deviations to SM predictions may still show up in total cross sections and distributions, due to the effect of virtual particles in the loops. It is, therefore, of paramount importance that we are able to provide very precise theoretical predictions which match the precision reached by experimental measurements at the LHC.

Precision calculations for LHC observables require the evaluation of Feynman diagrams where additional virtual or

\footnotetext{
*jbroedel@physik.hu-berlin.de

claude.duhr@cern.ch

*dulatf@slac.stanford.edu

§lorenzo.tancredi@cern.ch
}

Published by the American Physical Society under the terms of the Creative Commons Attribution 4.0 International license. Further distribution of this work must maintain attribution to the author(s) and the published article's title, journal citation, and DOI. Funded by SCOAP ${ }^{3}$. real particles are present in the process. While next-toleading order computations, which include contributions with one additional real or virtual particle, were fully automated a few years ago, we are currently living through an era where next-to-next-to-leading order computations are becoming the new standard. Key in this progress has been, among other things, an improved understanding of how to perform two-loop computations. In particular, it has become clear that large classes of Feynman integrals can be expressed in terms of a class of special functions known as multiple polylogarithms (MPLs) [1-3]. One of the main advantages of working with MPLs is that their underlying mathematical and algebraic structures are well understood [4,4-8].

It is well known, however, that not all two-loop Feynman integrals can be expressed in terms of MPLs alone. The first appearance of a two-loop integral that cannot be expressed in terms of MPLs goes back to QED [9], where it was observed that the electron self-energy involves integrals of elliptic type. Similar functions have recently shown up in two-loop computations relevant to LHC processes, like for example top-quark pair production [10-12], Higgs production in association with a jet [13] and top-mass effects in diphoton and dijet production [14]. The previous processes all explicitly involve a heavy massive particle in the loop, and it was assumed for a long time that massive propagators are a necessary condition for the appearance of nonpolylogarithmic structures. Recently, however, it was shown that elliptic integrals also show up in planar $\mathcal{N}=4$ Super Yang-Mills [15-17]. Although the previous 
examples make it clear that progress in multiloop computations will require a deeper understanding of Feynman integrals of elliptic type, still very little is known about the mathematical properties of the resulting class of functions. This, in turn, has sparked a lot of activity in recent years in trying to uncover the mathematics of elliptic Feynman integrals [12,18-33]

The most prominent elliptic Feynman integral is the socalled sunrise integral, i.e., the two-loop integral with three massive propagators. It had been observed already fifteen years ago that the maximal cut of this integral can be expressed in terms of complete elliptic integrals of the first kind [21]. The result for the full (uncut) integral, however, remained mysterious for more than a decade. In a landmark paper, Bloch and Vanhove have shown that the sunrise integral with three equal masses in two dimensions can naturally be written in terms of a generalization of the dilogarithm to an elliptic curve [23]. The latter is a special case of a more general class of functions, called elliptic multiple polylogarithms (eMPLs) [34-36], and they have recently appeared also in the context of superstring amplitudes at one loop [37-39]. The result of Ref. [23] has sparked a wealth of new results and representations for the sunrise integral, including also higher-order terms in dimensional regularization and results in four spacetime dimensions [12,24-31]. A common feature of these results is that most of them require the introduction of a new elliptic generalization of MPLs, whose relationship to the eMPLs that have appeared in pure mathematics and string theory is often unclear. This is somewhat disconcerting, because in the nonelliptic case it was precisely the realization that ordinary MPLs constitute the right class of functions with beautiful algebraic properties that was at the heart of a lot of progress in multiloop computations.

In the present paper, we try to close this gap, and we introduce a class of functions that are defined as iterated integrals on an elliptic curve. The ensuing functions have at most logarithmic singularities-thereby constituting a genuine generalization of polylogarithms to elliptic curves. We discuss how one can easily compute the sunrise integral in term of these functions, and we present analytic results for all the master integrals of the sunrise topology in $d=2-2 \epsilon$ dimensions. In particular, we present for the first time an analytic expression for the second master integral in the case of three unequal masses. In a companion paper [40], we study in detail some of the properties of our functions. In particular, we show that they are equivalent to the eMPLs introduced in the mathematics literature. As such, our functions genuinely deserve being called elliptic multiple polylogarithms as well. At the same time, this shows how the sunrise integral is connected to the eMPLs that have appeared in mathematics and string theory.

The outline of the paper is as follows: after providing a lightning overview of some background on the sunrise integrals in Sec. II, we will jump into the evaluation of the first master integral for the equal-mass sunrise integral in Sec. III. This integral will serve as our prime example of how eMPLs naturally arise in the context of the sunrise integrals. After this first encounter with iterated integrals on elliptic curves, we will discuss and compute the second master integral for the equal-mass sunrise integral in Sec. IV. We will collect structural results of the first sections, including the complete set of integration kernels that define eMPLs, in a brief summary Sec. V. In Sec. VI, we will apply our new language to the more complex scenario of sunrise integrals with three different masses. In particular, we will discuss the unitary cut of the sunrise integral as well as the unequal-mass master integrals for the sunrise topology from dispersion relations. In Sec. VII, we draw our conclusion.

\section{THE SUNRISE INTEGRAL: OVERVIEW}

The most popular example of a family of Feynman integrals that cannot be computed in terms of multiple polylogarithms are the sunrise integrals, which have received a lot of attention over the last few years. The sunrise integrals can be represented by the following graph,

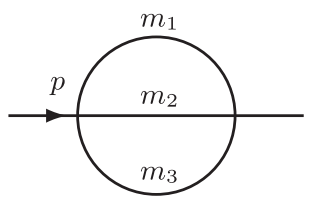

and the corresponding family of Feynman integrals reads

$$
\begin{aligned}
& S_{\nu_{1} \nu_{2} \nu_{3}}\left(S, m_{1}^{2}, m_{2}^{2}, m_{3}^{2}\right) \\
& \quad=\int \frac{\mathfrak{D}^{d} k_{1} \mathfrak{D}^{d} k_{2}}{\left(k_{1}^{2}-m_{1}^{2}\right)^{\nu_{1}}\left(k_{2}^{2}-m_{2}^{2}\right)^{\nu_{2}}\left(\left(k_{1}-k_{2}+p\right)^{2}-m_{3}^{2}\right)^{\nu_{3}}},
\end{aligned}
$$

where the integration measure is defined as

$$
\int \mathfrak{D}^{d} k \equiv e^{\gamma_{E} \epsilon} \int \frac{\mathrm{d}^{d} k}{i \pi^{d / 2}},
$$

$\gamma_{E}=-\Gamma^{\prime}(1)$ is the Euler-Mascheroni constant and $\nu_{i} \in \mathbb{Z}$ denote the multiplicities of the propagators. We work in dimensional regularization in $d=d_{0}-2 \epsilon$ dimensions, where $d_{0}$ is even. We define $S=-s=-p^{2}$, and the quantities $k_{i}$ and $m_{i}$ denote the loop momenta and the masses of the propagators respectively.

In terms of Feynman parameters, the integral in Eq. (2.1) can equivalently be written as

$$
\begin{aligned}
S_{\nu_{1} \nu_{2} \nu_{3}} & \left(S, m_{1}^{2}, m_{2}^{2}, m_{3}^{2}\right) \\
= & \frac{e^{2 \gamma_{E} \epsilon} \Gamma(\nu-d)(-1)^{\nu}}{\Gamma\left(\nu_{1}\right) \Gamma\left(\nu_{2}\right) \Gamma\left(\nu_{3}\right)} \int_{0}^{\infty} d x_{1} d x_{2} d x_{3} x_{1}^{\nu_{1}-1} x_{2}^{\nu_{2}-1} x_{3}^{\nu_{3}-1} \\
& \times \frac{\mathcal{U}^{\nu-3 / 2 d}}{\mathcal{F}^{\nu-d}} \delta\left(1-x_{3}\right)
\end{aligned}
$$


with $\nu \equiv \sum_{i=1}^{3} \nu_{i}$, and the Symanzik polynomials are

$$
\begin{aligned}
& \mathcal{U}=x_{1} x_{2}+x_{2} x_{3}+x_{1} x_{3} \text { and } \\
& \mathcal{F}=x_{1} x_{2} x_{3} S+\left(m_{1}^{2} x_{1}+m_{2}^{2} x_{2}+m_{3}^{2} x_{3}\right) \mathcal{U}
\end{aligned}
$$

In order to get a feeling for the class of functions that show up when evaluating the integral in Eq. (2.3), let us first consider the case where all masses are equal, $m_{i}=m \neq 0$. We will return to the unequal-mass case in Sec. VI. Throughout this paper, we only discuss the sunrise integrals in $d=2-2 \epsilon$ dimensions. This allows us to focus on the elliptic core of the integrals. We stress that the restriction to two dimensions is not a limitation, because the results in $d=4-2 \epsilon$ dimensions can always be recovered via dimensional recurrence relations $[41,42]$.

Equation (2.1) defines an infinite family of Feynman integrals, but not all members of the family are independent. We can use integration-by-parts (IBP) identities to reduce any member in the sunrise topology with three equal (nonzero) masses to a linear combination of three master integrals, which we can choose to be $S_{111}, S_{211}$ and $S_{110}$. $S_{110}$ is a product of one-loop integrals and will not be discussed any further. Note that, of course, in order to obtain an integral family for the sunrise graph closed under IBP identities we need to add two independent scalar products to the definition in Eq. (2.1). In the following we will often refer to $S_{111}$ and $S_{211}$ loosely as the first and second master integrals. These two master integrals will be our guiding examples for exploring the world of elliptic multiple polylogarithms in this article. We stress that our paper is not the first to consider the sunrise integrals, but they have been computed in various different guises in terms of functions that cannot be expressed in terms of multiple polylogarithms (MPLs). The goal of this paper is to show that there is a natural class of iterated integrals on an elliptic curve through which our results can be expressed. This class of iterated integrals, whose mathematical properties are spelled out in more detail in a companion paper [40], have at most logarithmic singularities on the elliptic curve, and as such they deserve to be called elliptic multiple polylogarithms (eMPLs). Moreover, in Ref. [40] we show that these elliptic polylogarithms are indeed very closely related to the multiple elliptic polylogarithms considered in the mathematics literature [34-36].

In the next two sections, we will describe in detail the computation of the first and second master integrals.

\section{FIRST MASTER INTEGRAL OF THE EQUAL-MASS SUNRISE}

\section{A. The first master integral in $\boldsymbol{d}=\mathbf{2}$ dimensions}

Starting from Eq. (2.3), the first master integral of the equal-mass sunrise topology admits the integral representation

$$
\begin{aligned}
S_{111}\left(S, m^{2}\right) \equiv & S_{111}\left(S, m^{2}, m^{2}, m^{2}\right) \\
=- & \Gamma(3-d) e^{2 \gamma_{E} \epsilon} \int_{0}^{\infty} d x_{1} d x_{2} d x_{3} \delta\left(1-x_{3}\right) \\
& \times \frac{\mathcal{U}^{-3 / 2(d-2)}}{\mathcal{F}^{3-d}} .
\end{aligned}
$$

The integral is convergent in two dimensions, and so we can simply perform the expansion in the dimensional regulator $\epsilon$ at the integrand level. At leading order in $\epsilon$, the integral $S_{111}$ is determined solely by the polynomial $\mathcal{F}$ defined in Eq. (2.4):

$$
\begin{aligned}
& \left.S_{111}\left(S, m^{2}\right)\right|_{\epsilon^{0}} \\
& \quad=-\int_{0}^{\infty} d x_{1} d x_{2} \frac{1}{m^{2}\left(x_{1}+x_{2}+1\right) s_{2}\left(x_{1}, x_{2}, 1\right)+S x_{1} x_{2}} .
\end{aligned}
$$

The denominator is a quadratic polynomial in $x_{2}$. We perform the change of variables $x_{1}=x /(1-x)$, and we find

$$
\begin{aligned}
& \left.S_{111}\left(S, m^{2}\right)\right|_{\epsilon^{0}} \\
& =-\int_{0}^{1} d x \int_{0}^{\infty} d x_{2} \frac{1}{m^{2}\left(x+x_{2}\right)\left(1+(1-x) x_{2}\right)+S(1-x) x x_{2}} .
\end{aligned}
$$

After linearizing the denominator by partial fractioning in $x_{2}$, the remaining integral is easily performed, yielding

$$
\left.S_{111}\left(S, m^{2}\right)\right|_{\epsilon^{0}}=\frac{1}{\left(m^{2}+S\right)} \int_{0}^{1} d x \frac{\log \chi}{y},
$$

with

$$
\chi=\frac{x(x-1)\left(m^{2}+S\right)-m^{2}-\left(m^{2}+S\right) y}{x(x-1)\left(m^{2}+S\right)-m^{2}+\left(m^{2}+S\right) y},
$$

and

$$
y=\sqrt{\left(x-a_{1}\right)\left(x-a_{2}\right)\left(x-a_{3}\right)\left(x-a_{4}\right)},
$$

where $a_{i}, 1 \leq i \leq 4$, denote the roots of the quartic polynomial inside the square root

$$
\begin{array}{ll}
a_{1}=\frac{1}{2}(1-\sqrt{1+\rho}), & a_{2}=\frac{1}{2}(1+\sqrt{1+\rho}), \\
a_{3}=\frac{1}{2}(1-\sqrt{1+\bar{\rho}}), & a_{4}=\frac{1}{2}(1+\sqrt{1+\bar{\rho}}) .
\end{array}
$$

The auxiliary variables $\rho$ and $\bar{\rho}$ are defined by

$$
\rho=-\frac{4 m^{2}}{(m+\sqrt{-S})^{2}} \quad \text { and } \quad \bar{\rho}=-\frac{4 m^{2}}{(m-\sqrt{-S})^{2}} .
$$

For real values of $\sqrt{-S}$ and $m$ they are complex conjugates of each other. The four roots $a_{i}$ of the quartic polynomial are 
clearly distinct for distinct and nonvanishing values of $\sqrt{-S}$ and $m$, and therefore the quartic polynomial is irreducible.

In absence of the logarithm in the numerator in Eq. (3.4), the integral would evaluate to an incomplete elliptic integral of the first kind:

$$
\mathrm{F}\left(x \mid w^{2}\right)=\int_{0}^{x} \frac{d t}{\sqrt{\left(1-t^{2}\right)\left(1-w^{2} t^{2}\right)}} .
$$

While elliptic integrals of this type are well studied in 19th century mathematics, integrals of the type in Eq. (3.4), with a logarithm in the numerator, are not part of the classical literature on elliptic integrals; only recently a subclass of these integrals has been studied in detail in Ref. [31]. One of the main goals of this paper is to show how such integrals can be performed in complete generality in terms of a welldefined class of iterated integrals.

We start by analyzing the logarithmic term in Eq. (3.4) separately. Differentiating with respect to $x$ and integrating back, we find the following integral representation for the logarithm,

$\log \chi=\int_{0}^{x} \frac{d x^{\prime}}{y_{x^{\prime}}}\left[2 x^{\prime}-1-\frac{m^{2}}{\left(m^{2}+S\right)\left(1-x^{\prime}\right)}+\frac{m^{2}}{\left(m^{2}+S\right) x^{\prime}}\right]$.

Perhaps surprisingly, the logarithm itself can be written as an integral of elliptic type, as indicated by $y_{x^{\prime}}$ in the denominator (which denotes $y$ evaluated at $x^{\prime}$ ). Concretely, Eq. (3.10) contains three integrals of elliptic type:

$$
\int \frac{d x}{y}, \quad \int \frac{d x}{y} x, \quad \int \frac{d x}{y} \frac{1}{x-c} .
$$

The first integral in Eq. (3.11) bears some similarities with the elliptic integral of the first kind $\mathrm{K}$ in Eq. (3.9). One is tempted to try to integrate the remaining two integrals by parts in order to reduce them to those of the first kind. That attempt is futile, however, and one can show that indeed the three integrals in Eq. (3.11) are independent with respect to IBP identities. This, in turn, is a well-known result in mathematics: the second and third integral in Eq. (3.11) are related to the incomplete elliptic integrals of third kind:

$\Pi\left(n^{2}, x \mid w^{2}\right)=\int_{0}^{x} \frac{d t}{\sqrt{\left(1-t^{2}\right)\left(1-w^{2} t^{2}\right)}} \frac{1}{1-n^{2} t^{2}}$.

As a conclusion, all the integrations in Eq. (3.10) can be performed in terms of elliptic integrals of the first and third kind, leading to an intriguing relation between a logarithm and a sum of elliptic integrals.

When we rewrite the logarithm in Eq. (3.4) as a combination of elliptic integrals, the classical theory of elliptic integrals of the 19th century is no longer sufficient to perform the integral. Very loosely speaking, our goal is to extend the theory of elliptic integrals to include those iterated integrations. This is achieved by defining an elliptic generalization of multiple polylogarithms, elliptic multiple polylogarithms (eMPLs), which are defined as iterated integrals over suitable integration kernels $\psi_{i}$,

$$
\mathrm{E}_{4}\left(\begin{array}{l}
n_{1} \ldots n_{k} \\
c_{1} \ldots c_{k}
\end{array} ; x\right)=\int_{0}^{x} d t \psi_{n_{1}}\left(c_{1}, t\right) \mathrm{E}_{4}\left(\begin{array}{l}
n_{2} \ldots n_{k} \\
c_{2} \ldots c_{k}
\end{array} ; t\right),
$$

where $n_{i} \in \mathbb{Z}, c_{i} \in \mathbb{C} \cup\{\infty\}$ and the recursion starts with $E_{4}(; x)=1$. Note that the definition in Eq. (3.13) is in complete analogy with the ordinary multiple polylogarithms, which are defined recursively by

$$
G\left(a_{1}, \ldots, a_{n} ; x\right)=\int_{0}^{x} \frac{d x^{\prime}}{x^{\prime}-a_{1}} G\left(a_{2}, \ldots, a_{n} ; x^{\prime}\right),
$$

starting with $G(; x)=1$.

Let us briefly discuss some of the properties of the integration kernels $\psi_{i}$ in Eq. (3.13). The kernels should satisfy three basic requirements. First, they should not be total derivatives, because otherwise the integration would be trivial. Second, to form a good basis, they should be independent with respect to IBP identities. Finally, since our eMPLs should have at most logarithmic singularities, the integration kernels should have at most simple poles. From the previous discussion, it follows that we have natural candidates in Eq. (3.11) that satisfy all of the requirements. We therefore define the following integration kernels,

$$
\begin{aligned}
\psi_{0}(0, x) & =\frac{c_{4}}{y}, \quad \psi_{-1}(\infty, x)=\frac{x}{y}, \\
\psi_{-1}(c, x) & =\frac{y_{c}}{(x-c) y}-\frac{\delta_{c 0}}{x},
\end{aligned}
$$

where the kernel $\psi_{0}(0, x)$ includes the normalization factor $c_{4}=\frac{1}{2} \sqrt{a_{13} a_{24}}$, with $a_{i j}=a_{i}-a_{j}$. Since our iterated integrals shall be a generalization of ordinary MPLs, we include their integration kernel from Eq. (3.14), and we define

$$
\psi_{1}(c, x)=\frac{1}{x-c} .
$$

Note that the previous equation implies that ordinary MPLs are just a subset of eMPLs. While these definitions may seem $a d$ hoc at this point, we will argue in more detail in Sec. IV that these kernels indeed define genuine eMPLs. In particular, we will see that we need to extend the set of kernels in Eq. (3.15) in order to obtain a complete and independent set of eMPLs. The kernels in Eq. (3.15), however, are sufficient for the computation of the first master integral of the equal-mass sunrise topology, and so we defer a more detailed discussion of the complete set of integration kernels to a subsequent section.

Let us also comment on the singularity structure of the integration kernels $\psi_{i}$. Different types of kernels are indexed by an integer subscript, and the first argument of $\psi_{i}(c, x)$ indicates that this kernel has a simple pole at $x=c$. In particular, $c$ may be infinite, indicating that there is a pole at $x=\infty$. Indeed, letting $x=1 / u$, we see that $\psi_{-1}(\infty, x)$ has a pole at $x=\infty$ (that shows up as a pole at 
$u=0$ ). The only kernel deviating from this nomenclature is $\psi_{0}(0, x)$, which does not have any pole. Further clarification is required for the kernel $\psi_{-1}(0, x)$ : it is straightforward to check that $1 /(x y)$ has a pole $x=0$ (assuming $a_{i} \neq 0$ ), leading to an end-point singularity in Eq. (3.13) whenever $c_{k}=0$. Correspondingly, the pole at $x=0$ is removed by the Kronecker $\delta$ term, making the convergence of the integral manifest. Note that also $\psi_{1}(0, x)=1 / x$ is singular at $x=0$. In this case, however, we do not subtract the singularity, but we use the following special definition, well-known from the case of ordinary MPLs,

$$
\begin{gathered}
\mathrm{E}_{4}(\underbrace{\underbrace{1 \ldots 1}_{1 \ldots 0}}_{n \text { times }} ; x)=G(\underbrace{0 \ldots 0}_{n \text { times }} ; x)=\frac{1}{n !} \log ^{n} x, \\
\log x=\int_{1}^{x} \frac{d x^{\prime}}{x} .
\end{gathered}
$$

Finally, by using $y$ in Eq. (3.15), we are implicitly using information about the geometry of the underlying elliptic curve we are working on. Thus it would be accurate to attach the root vector $\vec{a}=\left(a_{1}, a_{2}, a_{3}, a_{4}\right)$ as an additional parameter to each of the kernels and $\mathrm{E}_{4}$. However, in order to avoid cluttering the notation, we always suppress the dependence on the root vector, as in this paper all our equations will involve objects defined on the same elliptic curve only (we assume the external kinematics fixed).

Armed with these definitions, we can now return to Eq. (3.10) and write it in terms of the integration kernels,

$$
\begin{aligned}
\log \chi= & \int_{0}^{x} d x^{\prime}\left[\frac{1}{c_{4}} \psi_{0}\left(0, x^{\prime}\right)-2 \psi_{-1}\left(\infty, x^{\prime}\right)\right. \\
& \left.+\psi_{-1}\left(0, x^{\prime}\right)+\psi_{1}\left(0, x^{\prime}\right)+\psi_{-1}\left(1, x^{\prime}\right)\right] .
\end{aligned}
$$

Using the iterated definition of the eMPLs in Eq. (3.13), all integrations can be performed with ease and yield

$$
\begin{aligned}
\log \chi= & \frac{1}{c_{4}} \mathrm{E}_{4}\left(\begin{array}{l}
0 \\
0
\end{array} x\right)-2 \mathrm{E}_{4}\left(\begin{array}{c}
-1 \\
\infty
\end{array} ; x\right)+\mathrm{E}_{4}\left(\begin{array}{c}
-1 \\
0
\end{array} ; x\right) \\
& +\mathrm{E}_{4}\left(\begin{array}{l}
1 \\
0
\end{array} ; x\right)+\mathrm{E}_{4}\left(\begin{array}{c}
-1 \\
1
\end{array} ; x\right) .
\end{aligned}
$$

The previous equation is a remarkable identity that allows us to write a logarithm with a complicated algebraic argument in terms of elliptic polylogarithms with simple arguments. Inserting Eq. (3.19) into Eq. (3.4), we obtain

$$
\begin{aligned}
\left.S_{111}\left(S, m^{2}\right)\right|_{\epsilon^{0}}= & \frac{1}{\left(m^{2}+S\right) c_{4}} \int_{0}^{1} d x \psi_{0}(0, x)\left[\frac{1}{c_{4}} \mathrm{E}_{4}\left(\begin{array}{l}
0 \\
0
\end{array} x\right)\right. \\
& -2 \mathrm{E}_{4}\left(\begin{array}{c}
-1 \\
\infty
\end{array} ; x\right)+\mathrm{E}_{4}\left(\begin{array}{c}
-1 \\
0
\end{array} ; x\right) \\
& \left.+\mathrm{E}_{4}\left(\begin{array}{l}
1 \\
0
\end{array} ; x\right)+\mathrm{E}_{4}\left(\begin{array}{c}
-1 \\
1
\end{array} ; x\right)\right]
\end{aligned}
$$

where we have replaced $1 / y$ with the integral kernel $\psi_{0}(0, x) / c_{4}$. The remaining integrations can easily be performed using Eq. (3.13), which leads to the result

$$
\begin{aligned}
\left.S_{111}\left(S, m^{2}\right)\right|_{\epsilon^{0}}= & \frac{1}{\left(m^{2}+S\right) c_{4}}\left[\frac{1}{c_{4}} \mathrm{E}_{4}\left(\begin{array}{cc}
0 & 0 \\
0 & 0
\end{array} 1\right)-2 \mathrm{E}_{4}\left(\begin{array}{cc}
0 & -1 \\
0 & \infty
\end{array} ; 1\right)\right. \\
& \left.+\mathrm{E}_{4}\left(\begin{array}{ccc}
0 & -1 \\
0 & 0
\end{array} ; 1\right)+\mathrm{E}_{4}\left(\begin{array}{cc}
0 & -1 \\
0 & 1
\end{array} ; 1\right)-\mathrm{E}_{4}\left(\begin{array}{cc}
1 & 0 \\
0 & 0
\end{array} 1\right)\right] .
\end{aligned}
$$

Equation (3.21) is the final result for the first master integral of the sunrise topology in $d=2$ dimensions. As promised, we see that the result can be cast entirely in the form of a linear combination of eMPLs defined in Eq. (3.13). Before we continue and discuss how to extend our framework to higher orders in $\epsilon$ and the second master integral, let us summarize our main steps: we were able to write a simple function, the logarithm appearing in Eq. (3.4), with complicated arguments involving a square root of a quartic polynomial, in terms of more complicated functions, the elliptic multiple polylogarithms, with a simple argument. This strategy is reminiscent of the strategy employed successfully in the computation of polylogarithmic integrals. A key ingredient in establishing our method was the identification of the integration kernels $\psi_{i}$. The integral considered so far was simple enough to appear directly in terms of these kernels. However, as will be discussed below, it is possible to tackle more complicated integrals using IBP identities.

\section{B. The first master integral in dimensional regularization}

So far we have only discussed the master integral $S_{111}$ in strictly two dimensions. In the remainder of this section, we extend our analysis to higher orders in the $\epsilon$ expansion, and we show that $S_{111}$ can be expressed in terms of eMPLs at every order in dimensional regularization. We only discuss the linear term in $\epsilon$ explicitly. The extension to higher orders is straightforward.

Expanding the Feynman parameter integral in Eq. (3.1) to linear order in $\epsilon$, we find,

$$
\begin{aligned}
\left.S_{111}\left(S, m^{2}\right)\right|_{\epsilon^{1}}= & \int_{0}^{\infty} d x_{1} d x_{2} d x_{3} \\
& \times \frac{\delta\left(1-x_{3}\right)[2 \log (\mathcal{F})-3 \log (\mathcal{U})]}{\mathcal{F}} .
\end{aligned}
$$

The denominator in Eq. (3.22) is identical to the denominator in Eq. (3.2), and so we can expect that the integration can be done using a similar algorithm as for $\epsilon=0$. Hence, we let $x_{1}=x /(1-x)$, and the result of the integral over $x_{2}$ can be written in terms of ordinary multiple polylogarithms as 


$$
\begin{aligned}
\left.S_{111}\left(S, m^{2}\right)\right|_{\epsilon^{1}}= & \frac{1}{m^{2}+S} \int_{0}^{1} \frac{d x}{y}\left[G(0 ; \chi)\left(G\left(0 ; \chi^{\prime}\right)+G(1 ; x)+4 G(1 ; \chi)+2 G\left(0 ; m^{2}\right)-3 G\left(\frac{\chi^{\prime}}{\chi} ; x\right)\right)\right. \\
& \left.-3 G(0,0 ; \chi)+3 G\left(0 ; \chi^{\prime}\right) G\left(\frac{\chi^{\prime}}{\chi} ; x\right)-3 G\left(\frac{\chi^{\prime}}{\chi}, 0 ; x\right)-4 G(0,1 ; \chi)-G\left(0 ; \chi^{\prime}\right) G\left(\chi^{\prime} ; x\right)+3 G\left(\chi^{\prime}, 0 ; x\right)-4 \zeta_{2}\right]
\end{aligned}
$$

where $\chi$ has been defined in Eq. (3.5), and we have introduced the quantity

$\chi^{\prime}=\frac{\left(m^{2}+S\right) y+m^{2}\left(x^{2}-x-1\right)+S(x-1) x}{2 m^{2}(x-1)}$.

The remaining integration over $x$ can be done following the same steps as for $\epsilon=0$ : we write every ordinary MPL with a complicated argument in the integrand as a linear combination of eMPLs such that the integration can immediately be performed using the definition of the $\mathrm{E}_{4}$ functions in Eq. (3.13). This rewriting can be done recursively in the weight of the MPLs. For example, let us consider how to rewrite $G(1 ; \chi)$ in terms of eMPLs. We can differentiate with respect to $x$ and integrate back, and we find,

$$
\begin{aligned}
G(1 ; \chi)= & -i \pi+\int_{0}^{x} \frac{d x^{\prime}}{y_{x^{\prime}}} \frac{\left(1-2 x^{\prime}\right)\left(m^{2}\left(1-\left(1-x^{\prime}\right) x^{\prime}\right)-S\left(1-x^{\prime}\right) x^{\prime}\right)}{2\left(1-x^{\prime}\right) x^{\prime}\left(m^{2}+S\right)}-\int_{0}^{x} d x^{\prime} \frac{1-2 x^{\prime}}{2 x^{\prime}\left(1-x^{\prime}\right)} \\
& +\sum_{i=1}^{4} \int_{0}^{x} \frac{d x^{\prime}}{x^{\prime}-a_{i}} \frac{\left(1-2 a_{i}\right)\left(\left(1-\left(1-a_{i}\right) a_{i}\right) m^{4}-\left(2\left(1-a_{i}\right) a_{i}+1\right) m^{2} S-\left(1-a_{i}\right) a_{i} S^{2}\right)}{\left(m^{2}+S\right)^{2} \prod_{j=1, j \neq k}^{4} a_{i j}} \\
= & -i \pi+\frac{1}{2}\left[2 \mathrm{E}_{4}\left(\begin{array}{c}
-1 \\
\infty
\end{array} ; x\right)-\frac{1}{c_{4}} \mathrm{E}_{4}\left(\begin{array}{c}
0 \\
0
\end{array} ; x\right)-\mathrm{E}_{4}\left(\begin{array}{c}
-1 \\
0
\end{array} ; x\right)-\mathrm{E}_{4}\left(\begin{array}{c}
-1 \\
1
\end{array} ; x\right)-2 \mathrm{E}_{4}\left(\begin{array}{c}
1 \\
0
\end{array} ; x\right)-\mathrm{E}_{4}\left(\begin{array}{c}
1 \\
1
\end{array} ; x\right)+\sum_{i=1}^{4} \mathrm{E}_{4}\left(\begin{array}{c}
1 \\
a_{i}
\end{array} ; x\right)\right] .
\end{aligned}
$$

We apply this procedure to every MPL of weight one. Next, we differentiate MPLs of weight two. The derivative contains MPLs of weight one, which we know how to express in terms of eMPLs. For example, we find,

$$
G(0,1 ; \chi)=-2 \zeta_{2}+\int_{0}^{x} d x^{\prime} \frac{d \chi_{x^{\prime}}}{d x^{\prime}} \frac{G\left(1 ; \chi_{x^{\prime}}\right)}{\chi_{x^{\prime}}}
$$

Inserting Eq. (3.25) into the integrand, we find

$$
\begin{aligned}
& G(0,1 ; \chi)=\frac{1}{2 c_{4}^{2}} \mathrm{E}_{4}\left(\begin{array}{cc}
0 & 0 \\
0 & 0
\end{array} ; x\right)+\frac{1}{c_{4}}\left[\frac{1}{2} \mathrm{E}_{4}\left(\begin{array}{cc}
-1 & 0 \\
0 & 0
\end{array} ; x\right)+\frac{1}{2} \mathrm{E}_{4}\left(\begin{array}{cc}
-1 & 0 \\
1 & 0
\end{array} ; x\right)-\mathrm{E}_{4}\left(\begin{array}{cc}
-1 & 0 \\
\infty & 0
\end{array} ; x\right)+\frac{1}{2} \mathrm{E}_{4}\left(\begin{array}{cc}
0 & -1 \\
0 & 0
\end{array} ; x\right)\right. \\
& +\frac{1}{2} \mathrm{E}_{4}\left(\begin{array}{cc}
0 & -1 \\
0 & 1
\end{array} ; x\right)-\mathrm{E}_{4}\left(\begin{array}{cc}
0 & -1 \\
0 & \infty
\end{array} ; x\right)+\frac{1}{2} \mathrm{E}_{4}\left(\begin{array}{ll}
0 & 1 \\
0 & 1
\end{array} ; x\right)-\frac{1}{2} \mathrm{E}_{4}\left(\begin{array}{ccc}
0 & 1 \\
0 & a_{1}
\end{array} ; x\right)-\frac{1}{2} \mathrm{E}_{4}\left(\begin{array}{lll}
0 & 1 \\
0 & a_{2}
\end{array} ; x\right) \\
& \left.-\frac{1}{2} \mathrm{E}_{4}\left(\begin{array}{cc}
0 & 1 \\
0 & a_{3}
\end{array} ; x\right)-\frac{1}{2} \mathrm{E}_{4}\left(\begin{array}{cc}
0 & 1 \\
0 & a_{4}
\end{array} ; x\right)-\frac{1}{2} \mathrm{E}_{4}\left(\begin{array}{ll}
1 & 0 \\
0 & 0
\end{array} ; x\right)+(\log x-i \pi) \mathrm{E}_{4}\left(\begin{array}{l}
0 \\
0
\end{array} ; x\right)\right]+\frac{1}{2} \mathrm{E}_{4}\left(\begin{array}{cc}
-1 & -1 \\
0 & 0
\end{array} ; x\right) \\
& +\frac{1}{2} \mathrm{E}_{4}\left(\begin{array}{cc}
-1 & -1 \\
0 & 1
\end{array} ; x\right)-\mathrm{E}_{4}\left(\begin{array}{cc}
-1 & -1 \\
0 & \infty
\end{array} ; x\right)+\frac{1}{2} \mathrm{E}_{4}\left(\begin{array}{cc}
-1 & 1 \\
0 & 1
\end{array} ; x\right)-\frac{1}{2} \mathrm{E}_{4}\left(\begin{array}{cc}
-1 & 1 \\
0 & a_{1}
\end{array} ; x\right)-\frac{1}{2} \mathrm{E}_{4}\left(\begin{array}{cc}
-1 & 1 \\
0 & a_{2}
\end{array} ; x\right) \\
& -\frac{1}{2} \mathrm{E}_{4}\left(\begin{array}{cc}
-1 & 1 \\
0 & a_{3}
\end{array} ; x\right)-\frac{1}{2} \mathrm{E}_{4}\left(\begin{array}{cc}
-1 & 1 \\
0 & a_{4}
\end{array} ; x\right)+\frac{1}{2} \mathrm{E}_{4}\left(\begin{array}{cc}
-1 & 1 \\
1 & 0
\end{array} ; x\right)+\frac{1}{2} \mathrm{E}_{4}\left(\begin{array}{cc}
-1 & -1 \\
1 & 1
\end{array} ; x\right)-\mathrm{E}_{4}\left(\begin{array}{cc}
-1 & -1 \\
1 & \infty
\end{array} ; x\right) \\
& +\frac{1}{2} \mathrm{E}_{4}\left(\begin{array}{cc}
-1 & 1 \\
1 & 1
\end{array} ; x\right)-\frac{1}{2} \mathrm{E}_{4}\left(\begin{array}{cc}
-1 & 1 \\
1 & a_{1}
\end{array} ; x\right)-\frac{1}{2} \mathrm{E}_{4}\left(\begin{array}{cc}
-1 & 1 \\
1 & a_{2}
\end{array} ; x\right)-\frac{1}{2} \mathrm{E}_{4}\left(\begin{array}{cc}
-1 & -1 \\
1 & a_{3}
\end{array} ; x\right)-\frac{1}{2} \mathrm{E}_{4}\left(\begin{array}{cc}
-1 & 1 \\
1 & a_{4}
\end{array} ; x\right) \\
& -\mathrm{E}_{4}\left(\begin{array}{cc}
-1 & -1 \\
\infty & 0
\end{array} ; x\right)-\mathrm{E}_{4}\left(\begin{array}{cc}
-1 & -1 \\
\infty & 1
\end{array} ; x\right)+2 \mathrm{E}_{4}\left(\begin{array}{cc}
-1 & -1 \\
\infty & \infty
\end{array} ; x\right)-\mathrm{E}_{4}\left(\begin{array}{cc}
-1 & 1 \\
\infty & 1
\end{array} ; x\right)+\mathrm{E}_{4}\left(\begin{array}{cc}
-1 & 1 \\
\infty & a_{1}
\end{array} ; x\right)
\end{aligned}
$$

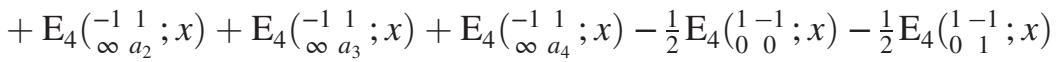

$$
\begin{aligned}
& +\mathrm{E}_{4}\left(\begin{array}{ll}
1 & -1 \\
0 & \infty
\end{array} ; x\right)+\frac{1}{2} \mathrm{E}_{4}\left(\begin{array}{ll}
1 & 1 \\
0 & 1
\end{array} ; x\right)-\frac{1}{2} \mathrm{E}_{4}\left(\begin{array}{cc}
1 & 1 \\
0 & a_{1}
\end{array} ; x\right)-\frac{1}{2} \mathrm{E}_{4}\left(\begin{array}{cc}
1 & 1 \\
0 & a_{2}
\end{array} ; x\right)-\frac{1}{2} \mathrm{E}_{4}\left(\begin{array}{lll}
1 & 1 \\
0 & a_{3}
\end{array} ; x\right) \\
& -\frac{1}{2} \mathrm{E}_{4}\left(\begin{array}{cc}
1 & 1 \\
0 & a_{4}
\end{array} ; x\right)-2(\log x-i \pi) \mathrm{E}_{4}\left(\begin{array}{c}
-1 \\
\infty
\end{array} ; x\right)-i \pi \log x+\frac{\log ^{2} x}{2}+(\log x-i \pi) \mathrm{E}_{4}\left(\begin{array}{c}
-1 \\
0
\end{array} ; x\right) \\
& +(\log x-i \pi) \mathrm{E}_{4}\left(\begin{array}{c}
-1 \\
1
\end{array} ; x\right)-2 \zeta_{2} \text {. }
\end{aligned}
$$


The same idea can be applied to every MPL in Eq. (3.23). After this is done, we can easily perform the integral over $x$ using the definition of eMPLs in Eq. (3.13). The final result reads

$$
\begin{aligned}
& \left.S_{111}\left(S, m^{2}\right)\right|_{\epsilon^{1}}=\frac{1}{\left(m^{2}+S\right) c_{4}}\left[\frac { 1 } { 2 } \operatorname { l o g } m ^ { 2 } \left(4 \mathrm{E}_{4}\left(\begin{array}{cc}
1 & 0 \\
0 & 0
\end{array} 1\right)-4 \mathrm{E}_{4}\left(\begin{array}{cc}
0-1 & 0
\end{array} ; 1\right)-4 \mathrm{E}_{4}\left(\begin{array}{cc}
0-1 \\
0 & 1
\end{array} ; 1\right)+8 \mathrm{E}_{4}\left(\begin{array}{cc}
0-1 \\
0 & \infty
\end{array} ; 1\right)\right.\right.
\end{aligned}
$$

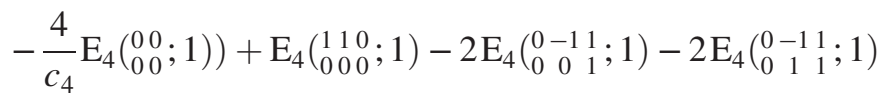

$$
\begin{aligned}
& -2 \mathrm{E}_{4}\left(\begin{array}{ccc}
0 & -1 & 1 \\
0 & \infty & 1
\end{array} ; 1\right)+5 \mathrm{E}_{4}\left(\begin{array}{ccc}
0 & 1 & -1 \\
0 & 0 & 0
\end{array} ; 1\right)+5 \mathrm{E}_{4}\left(\begin{array}{ccc}
0 & 1 & -1 \\
0 & 0 & 1
\end{array} ; 1\right)-4 \mathrm{E}_{4}\left(\begin{array}{ccc}
0 & 1 & -1 \\
0 & 0 & \infty
\end{array} ; 1\right)
\end{aligned}
$$

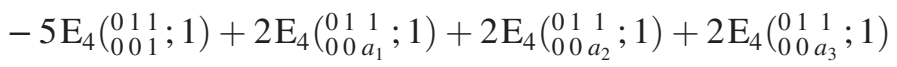

$$
\begin{aligned}
& +2 \mathrm{E}_{4}\left(\begin{array}{ccc}
0 & 1 & 1 \\
0 & 0 & a_{4}
\end{array} ; 1\right)+3 \mathrm{E}_{4}\left(\begin{array}{cccc}
0 & 1 & -1 \\
0 & 1 & 0
\end{array} ; 1\right)+3 \mathrm{E}_{4}\left(\begin{array}{cccc}
0 & 1 & -1 \\
0 & 1 & 1
\end{array} ; 1\right)-6 \mathrm{E}_{4}\left(\begin{array}{ccc}
0 & 1 & -1 \\
0 & 1 & \infty
\end{array} ; 1\right) \\
& -2 \mathrm{E}_{4}\left(\begin{array}{ccc}
0 & 1 & -1 \\
0 & a_{1} & 0
\end{array} ;\right)-2 \mathrm{E}_{4}\left(\begin{array}{ccc}
0 & 1 & -1 \\
0 & a_{1} & 1
\end{array} ; 1\right)+4 \mathrm{E}_{4}\left(\begin{array}{ccc}
0 & 1 & -1 \\
0 & a_{1} & \infty
\end{array} ; 1\right)-2 \mathrm{E}_{4}\left(\begin{array}{ccc}
0 & 1 & -1 \\
0 & a_{2} & 0
\end{array} ; 1\right) \\
& -2 \mathrm{E}_{4}\left(\begin{array}{ccc}
0 & 1 & -1 \\
0 & a_{2} & 1
\end{array} ; 1\right)+4 \mathrm{E}_{4}\left(\begin{array}{ccc}
0 & 1 & -1 \\
0 & a_{2} & \infty
\end{array} ; 1\right)-2 \mathrm{E}_{4}\left(\begin{array}{ccc}
0 & 1 & -1 \\
0 & a_{3} & 0
\end{array} ; 1\right)-2 \mathrm{E}_{4}\left(\begin{array}{ccc}
0 & 1 & -1 \\
0 & a_{3} & 1
\end{array} ; 1\right) \\
& +4 \mathrm{E}_{4}\left(\begin{array}{ccc}
0 & 1 & -1 \\
0 & a_{3} & \infty
\end{array} ; 1\right)-2 \mathrm{E}_{4}\left(\begin{array}{ccc}
0 & 1 & -1 \\
0 & a_{4} & 0
\end{array} ; 1\right)-2 \mathrm{E}_{4}\left(\begin{array}{ccc}
0 & 1 & -1 \\
0 & a_{4} & 1
\end{array} ; 1\right)+4 \mathrm{E}_{4}\left(\begin{array}{ccc}
0 & 1 & -1 \\
0 & a_{4} & \infty
\end{array} ; 1\right) \\
& +2 \mathrm{E}_{4}\left(\begin{array}{ccc}
1 & 0 & -1 \\
0 & 0 & 0
\end{array} ; 1\right)+2 \mathrm{E}_{4}\left(\begin{array}{ccc}
1 & 0 & -1 \\
0 & 0 & 1
\end{array} ; 1\right)+2 \mathrm{E}_{4}\left(\begin{array}{ccc}
1 & 0 & -1 \\
0 & 0 & \infty
\end{array} ; 1\right)-3 \mathrm{E}_{4}\left(\begin{array}{lll}
1 & 0 & 1 \\
0 & 0 & 1
\end{array} ; 1\right)
\end{aligned}
$$

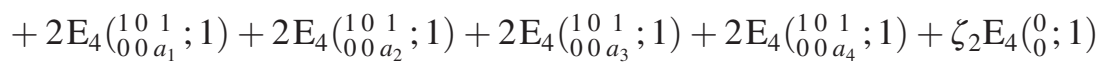

$$
\begin{aligned}
& +\frac{1}{c_{4}}\left(\mathrm{E}_{4}\left(\begin{array}{ccc}
0 & 0 & 1 \\
0 & 0 & 1
\end{array} ; 1\right)+2 \mathrm{E}_{4}\left(\begin{array}{cccc}
0 & 1 & 0 \\
0 & 0 & 0
\end{array} ; 1\right)+3 \mathrm{E}_{4}\left(\begin{array}{cccc}
0 & 1 & 0 \\
0 & 1 & 0
\end{array} ; 1\right)-2 \mathrm{E}_{4}\left(\begin{array}{ccc}
0 & 1 & 0 \\
0 & a_{1} & 0
\end{array} ; 1\right)\right. \\
& \left.\left.-2 \mathrm{E}_{4}\left(\begin{array}{ccc}
0 & 1 & 0 \\
0 & a_{2} & 0
\end{array} 1\right)-2 \mathrm{E}_{4}\left(\begin{array}{ccc}
0 & 1 & 0 \\
0 & a_{3} & 0
\end{array} ; 1\right)-2 \mathrm{E}_{4}\left(\begin{array}{ccc}
0 & 1 & 0 \\
0 & a_{4} & 0
\end{array} ; 1\right)-\mathrm{E}_{4}\left(\begin{array}{ccc}
1 & 0 & 0 \\
0 & 0 & 0
\end{array} ;\right)\right)\right] .
\end{aligned}
$$

We see that, just like for the leading term in the expansion, we can cast the result in the form of a linear combination of eMPLs. It is easy to extend the procedure used to perform the integration at higher orders in $\epsilon$, which proves that the $S_{111}$ in $d=2-2 \epsilon$ dimensions can be expressed in terms of $E_{4}$ functions at every order in dimensional regularization.

\section{THE SECOND MASTER INTEGRAL OF THE EQUAL-MASS SUNRISE}

In this section, we discuss the second master integral $S_{211}$ of the equal-mass sunrise topology. We will follow the same strategy as for the first master integral, i.e., we start from the Feynman parameter representation of $S_{211}$ and perform all the integrations sequentially in terms of eMPLs. In the process, we will discover that the integration kernels introduced in Eq. (3.15) are insufficient to perform all the integrals, and we will be forced to extend our set of integration kernels.

Let us start from the expression of the second master integral in terms of Feynman parameters:

$$
\begin{aligned}
S_{211}\left(S, m^{2}\right)= & \Gamma(2+2 \epsilon) e^{2 \gamma_{E} \epsilon} \\
& \times \int_{0}^{\infty} d x_{1} d x_{2} d x_{3} \delta\left(1-x_{3}\right) \frac{x_{1} \mathcal{U}^{1+3 \epsilon}}{\mathcal{F}^{2+2 \epsilon}},
\end{aligned}
$$

where the Symanzik-polynomials $\mathcal{F}$ and $\mathcal{U}$ have been defined in Eq. (2.4). The integral is finite in two dimensions, and the leading term in $\epsilon$ is

$\left.S_{211}\left(S, m^{2}\right)\right|_{\epsilon^{0}}=\int_{0}^{\infty} d x_{1} d x_{2} d x_{3} \frac{\delta\left(1-x_{3}\right) x_{1} s_{2}}{\left[m^{2}\left(x_{1}+x_{2}+x_{3}\right) s_{2}+S s_{3}\right]^{2}}$.

The previous integral is very similar in structure to the Feynman parameter integral representation of the first master integral in Eq. (3.2). The main difference is that we have an additional power of the polynomial $\mathcal{F}$ in the denominator and pick up numerator terms from the polynomial $\mathcal{U}$. These will lead to the appearance of rational prefactors in the integrand that need to be dealt with using integration-by-parts identities.

To start, we proceed in exactly the same way as for $S_{111}$ in the previous section. We eliminate the integration over $x_{3}$ using the $\delta$ function, and we map $x_{1}$ to the unit interval by changing variables according to $x_{1}=x /(1-x)$. Afterwards, we can factorize the quadratic polynomial in $x_{2}$ in the denominator and perform partial fractioning. The integral over $x_{2}$ can then be done using standard techniques. We find the following representation of $S_{211}$ :

$$
\left.S_{211}\left(S, m^{2}\right)\right|_{\epsilon^{0}}=\int_{0}^{1} d x\left(\mathcal{I}_{1}+\mathcal{I}_{2}\right)
$$

where 
$\mathcal{I}_{1}=\frac{x\left(m^{2}\left(1-x+x^{2}\right)-S(1-x) x\right)}{m^{2}\left(m^{2}+S\right)^{2}\left(x-a_{1}\right)\left(x-a_{2}\right)\left(x-a_{3}\right)\left(x-a_{4}\right)}$,

$\mathcal{I}_{2}=\frac{x\left(m^{2}\left(1-x+x^{2}\right)+S(1-x) x\right)}{\left(m^{2}+S\right)^{3}\left(x-a_{1}\right)\left(x-a_{2}\right)\left(x-a_{3}\right)\left(x-a_{4}\right) y} \log \chi$,

where $\chi$ and $y$ are defined in Eqs. (3.5) and (3.6). We will evaluate the integral in Eq. (4.3) by first computing a primitive of $\mathcal{I}_{i}$, and then we evaluate the primitives at the integration boundaries.

Let us begin by computing a primitive of $\mathcal{I}_{1}$. $\mathcal{I}_{1}$ does not contain any dependence on the square root $y$, and so we can simply compute the primitive in terms of ordinary MPLs after partial fractioning of the denominator. Since we want to combine the result with the elliptic contributions from $\mathcal{I}_{2}$, we find it convenient to write all ordinary MPLs from the beginning in terms of eMPLs. We find,

$$
\begin{aligned}
\int d x \mathcal{I}_{1}= & \sum_{k=1}^{4} \frac{a_{k}\left(\left(1-\left(1-a_{k}\right) a_{k}\right) m^{2}-\left(1-a_{k}\right) a_{k} S\right)}{m^{2}\left(m^{2}+S\right)^{2} \prod_{i=1, i \neq k}^{4} a_{k i}} \\
& \times \mathrm{E}_{4}\left(\begin{array}{c}
1 \\
a_{k}
\end{array} ; x\right) .
\end{aligned}
$$

Next we turn to the second term in Eq. (4.3). In contrast to $\mathcal{I}_{1}$, this term is of elliptic type due to the presence of $y$ in the denominator. Let us recall that $\log \chi$ can be cast in the form of a linear combination of eMPLs in Eq. (3.19). Partial fractioning in $x$ leads to integrals of the form

$$
\int d x \frac{\mathcal{X}(x)}{y\left(x-a_{i}\right)}
$$

where $\mathcal{X}(x)$ is some eMPL. At first glance, this integral looks very similar to Eq. (3.15) with $c=a_{i}$. There is however a crucial difference: the integral in Eq. (3.15) has a simple pole at $x=c$ for $c \neq a_{i}$. Since $x=a_{i}$ is a zero of the square root $y$, the integrand in Eq. (4.6) behaves like $\left(x-a_{i}\right)^{-3 / 2}$ for $x \sim a_{i}$. Hence, the point $x=a_{i}$ is not a pole in Eq. (4.6), but rather a branch point of the square root. In the next subsection, we discuss how to evaluate such integrals.

\section{A. Intermezzo: Integration of eMPLs with rational coefficients}

Let us begin by considering the simplest instance of the types of integrals encountered in Eq. (4.6), corresponding to $\mathcal{X}(x) \equiv 1$. Without loss of generality, we restrict the discussion to the case $i=1$. We find that we can write the integral in Eq. (4.6) as

$$
\begin{aligned}
\int \frac{d x}{y\left(x-a_{1}\right)}= & \frac{1}{a_{12} a_{13} a_{14}}\left[-\frac{2 y}{x-a_{1}}+\left(3 a_{1}-\bar{s}_{1}\right) \int \frac{d x}{y}\left(x-a_{1}\right)\right. \\
& \left.+2 \int \frac{d x}{y}\left(x-a_{1}\right)^{2}\right]
\end{aligned}
$$

with $\bar{s}_{n}=s_{n}\left(a_{2}, a_{3}, a_{4}\right)$ and $s_{n}$ are the symmetric polynomials of degree $n$. We can immediately recognize our integration kernels $\psi_{0}(0, x)$ and $\psi_{-1}(\infty, x)$ in the formula above. However, we also find the integral

$$
\int \frac{d x}{y} x^{2}
$$

which does not immediately translate into one of our kernels in Eq. (3.15), nor can it be reduced to them using IBP identities. We are therefore forced to introduce a new kernel, which we define by

$$
\tilde{\Phi}_{4}(x)=\frac{1}{c_{4} y}\left(x^{2}-\frac{s_{1}}{2} x+\frac{s_{2}}{6}\right) .
$$

This kernel contains not only the necessary $x^{2} / y$ term, but also terms proportional to $\psi_{-1}(\infty, x)$ and $\psi_{0}(0, x)$. These terms are purely conventional at this point.

The object in Eq. (4.9) seems rather random at first sight; in particular, we do not seem to be consistent in our notation for the integration kernels. There is a reason for not associating the name $\psi_{i}$ to this kernel: we demand of our kernels $\psi_{i}$ that they have at most simple poles. The above kernel $\tilde{\Phi}_{4}(x)$, however, has a double pole at $x=\infty$ : indeed, letting $x=1 / u$, we can see that

$$
d x \frac{x^{2}}{y}=-\frac{d u}{u^{2}}-\frac{s_{1} d u}{2 u}+\mathcal{O}\left(u^{0}\right)
$$

and consequently,

$$
d x \tilde{\Phi}_{4}(x)=-\frac{d u}{u^{2}}+\mathcal{O}\left(u^{0}\right) .
$$

Note that the term proportional to $x / y$ removes the simple pole at $u=0$, so that $\tilde{\Phi}_{4}(x)$ has a double pole at infinity with vanishing residue.

As a consequence, $\tilde{\Phi}_{4}(x)$ does not meet the fundamental criterion that our integration kernels should have at most simple poles. However, a function with a double pole gives rise to a primitive with a simple pole. We define a primitive of $\tilde{\Phi}_{4}$ by

$$
Z_{4}(x)=\int_{a_{1}}^{x} d x^{\prime}\left(\tilde{\Phi}_{4}(x)+4 c_{4} \frac{\eta_{1}}{\omega_{1}} \frac{1}{y}\right),
$$

where $\omega_{1}$ is the first of the two periods of the elliptic curve, defined as

$$
\begin{aligned}
& \omega_{1}=2 c_{4} \int_{a_{2}}^{a_{3}} \frac{d x}{y}=2 \mathrm{~K}(\lambda) \quad \text { and } \\
& \omega_{2}=2 c_{4} \int_{a_{1}}^{a_{2}} \frac{d x}{y}=2 i \mathrm{~K}(1-\lambda),
\end{aligned}
$$

with

$$
\lambda=\frac{a_{14} a_{23}}{a_{13} a_{24}} .
$$


In the previous equation, $\mathrm{K}(\lambda) \equiv \mathrm{F}(1 \mid \lambda)$ denotes the complete elliptic integral of the first kind. Similarly, one defines the quasiperiods of the elliptic curve by

$$
\begin{aligned}
& \eta_{1}=-\frac{1}{2} \int_{a_{2}}^{a_{3}} d x \tilde{\Phi}_{4}(x)=\mathrm{E}(\lambda)-\frac{2-\lambda}{3} \mathrm{~K}(\lambda), \\
& \eta_{2}=-\frac{1}{2} \int_{a_{1}}^{a_{2}} d x \tilde{\Phi}_{4}(x)=-i\left[\mathrm{E}(1-\lambda)-\frac{1+\lambda}{3} \mathrm{~K}(1-\lambda)\right],
\end{aligned}
$$

where $\mathrm{E}(\lambda) \equiv \mathrm{E}(1 \mid \lambda)$ denotes the elliptic integral of the second kind,

$$
\mathrm{E}\left(x \mid w^{2}\right)=\int_{0}^{x} d t \frac{1-w^{2} t^{2}}{\sqrt{\left(1-t^{2}\right)\left(1-w^{2} t^{2}\right)}} .
$$

Since $Z_{4}(x)$ is a primitive of a function with a double pole at infinity, $Z_{4}(x)$ itself only has a single pole at $x=\infty$. Consequently, we can use it to define new integration kernels for our elliptic multiple polylogarithms,

$$
\psi_{1}(\infty, x)=\frac{c_{4}}{y} Z_{4}(x) .
$$

Let us make an important comment about this kernel. Unlike the kernels considered in Eq. (3.15), the function $\psi_{1}(\infty, x)$ is not rational or algebraic, but it is itself an integral that defines a transcendental function. We see here one of the main differences between ordinary and elliptic polylogarithms: while ordinary MPLs only require integration kernels that are rational, this is no longer the case for elliptic polylogarithms. Indeed, the kernel in Eq. (4.17) cannot be reduced to any of the kernels we defined so far. In particular, it is independent of $\psi_{-1}(\infty, x)$, even though both kernels have a single pole at infinity.

The fact that $\psi_{1}(\infty, x)$ is transcendental has far-reaching implications. In particular, we will not be able to simplify powers of kernels or products with other kernels using partial fractioning. Instead, powers or product of kernels that involve $Z_{4}$ will define new independent kernels. For example, we can consider,

$$
\psi_{-2}(c, x)=\frac{y_{c}}{(x-c) y} Z_{4}(x) .
$$

Higher powers of $Z_{4}(x)$ will lead to further kernels $\psi_{ \pm n}$ with $n>2$. We obtain in this way an infinite tower of independent integration kernels, which can be constructed in the above fashion by taking combinations of powers of $Z_{4}(x)$ with elementary rational kernels. This may sound disconcerting at first sight: we set out to perform integrals over functions involving elliptic square roots in a closed form only to find that an infinite set of basis kernels is required. While there is no way around this fact in general, it turns out that for a given problem-concretely, a given number of integrations - only a finite number of kernels will ever contribute. In fact, for the problems discussed in this note the elementary kernels in Eq. (3.15), together with the kernels defined in Eqs. (4.17) and (4.18), are sufficient to perform all the integrals.

Finally, after this very long digression, we can return to the integral in Eq. (4.7), and we can rewrite the term $x^{2} / y$ in terms of $\tilde{\Phi}_{4}(x)$. We obtain

$$
\int \frac{d x}{y\left(x-a_{1}\right)}=\frac{2}{a_{12} a_{13} a_{14}}\left[\frac{y}{x-a_{1}}+\mathrm{E}_{4}\left(\begin{array}{l}
0 \\
0
\end{array} x\right)\left(\frac{3 a_{1}^{2}-2 a_{1} \bar{s}_{1}+\bar{s}_{2}}{6 c_{4}}+4 \frac{c_{4} \eta_{1}}{\omega_{1}}\right)+c_{4} Z_{4}(x)\right] .
$$

\section{B. Back to the second master integral}

After this intermezzo on the integration on elliptic curves, let us now return to the computation of the second master integral of the sunrise topology with equal masses. We had arrived at a point where we needed to perform integrals of the type in Eq. (4.6). For $\mathcal{X}(x) \equiv 1$, we can simply follow the reasoning of the previous section. In general, however, $\mathcal{X}(x)$ will be an elliptic polylogarithm. Fortunately, we can use IBP to reduce all appearing integrals to our extended set of integral kernels. We refer the reader to Appendix A for a more detailed discussion. Integrating the $\mathcal{I}_{2}$ term in Eq. (4.3) comes down to finding primitives like the following:

$$
\begin{aligned}
\int \frac{d x}{y\left(x-a_{1}\right)} \mathrm{E}_{4}\left({ }_{0}^{0} ; x\right)= & \frac{1}{a_{12} a_{13} a_{14}}\left[\frac{2 y \mathrm{E}_{4}\left({ }_{0}^{0} ; x\right)}{a_{1}-x}+2 \int \frac{d x}{x-a_{1}} y\left[\partial_{x} \mathrm{E}_{4}\left({ }_{0}^{0} ; x\right)\right]+\left(3 a_{1}-\bar{s}_{1}\right) \int \frac{d x}{y}\left(x-a_{1}\right) \mathrm{E}_{4}\left(\begin{array}{l}
0 \\
0
\end{array} x\right)\right. \\
& \left.+2 \int \frac{d x}{y}\left(x-a_{1}\right)^{2} \mathrm{E}_{4}\left({ }_{0}^{0} ; x\right)\right] .
\end{aligned}
$$

The integrals appearing on the right-hand-side can be expressed in terms of our kernels to yield 


$$
\begin{aligned}
& \int \frac{d x}{x-a_{1}} y\left[\partial_{x} \mathrm{E}_{4}\left(\begin{array}{l}
0 \\
0
\end{array} ; x\right)\right]=c_{4} \mathrm{E}_{4}\left(\begin{array}{l}
1 \\
a_{1}
\end{array} ; x\right), \\
& \int \frac{d x}{y}\left(x-a_{1}\right) \mathrm{E}_{4}\left(\begin{array}{c}
0 \\
0
\end{array} ; x\right)=\mathrm{E}_{4}\left(\begin{array}{c}
-10 \\
\infty
\end{array} ; x\right)-\frac{a_{1}}{c_{4}} \mathrm{E}_{4}\left(\begin{array}{c}
00 \\
00
\end{array} ; x\right), \\
& \int \frac{d x}{y}\left(x-a_{1}\right)^{2} \mathrm{E}_{4}\left({ }_{0}^{0} ; x\right)=c_{4} \mathrm{E}_{4}\left(\begin{array}{c}
1 \\
\infty
\end{array} ; x\right)+c_{4} Z_{4}(x) \mathrm{E}_{4}\left({ }_{0}^{0} ; x\right)-\frac{1}{2}\left(3 a_{1}-\bar{s}_{1}\right) \mathrm{E}_{4}\left({\underset{\infty}{0} 0}_{-10}^{10} ; x\right)+\left[\frac{6 a_{1}^{2}-a_{1} \bar{s}_{1}-\bar{s}_{2}}{6 c_{4}}-\frac{4 c_{4} \eta_{1}}{\omega_{1}}\right] \mathrm{E}_{4}\left(\begin{array}{c}
00 \\
00
\end{array} ; x\right) .
\end{aligned}
$$

In a similar fashion we can perform the remaining integrals contributing to the primitive of $\mathcal{I}_{2}$ that are listed in Appendix B. Combining them together with the primitive of $\mathcal{I}_{1}$ in Eq. (4.5), we obtain the primitive of Eq. (4.3):

$$
\begin{aligned}
& \mathcal{C}_{0} \int d x\left(\mathcal{I}_{1}+\mathcal{I}_{2}\right)=-c_{4} \mathrm{E}_{4}(\underset{0}{-2} ; x)-c_{4} \mathrm{E}_{4}\left(\frac{-2}{1} ; x\right)+2 c_{4} \mathrm{E}_{4}(\underset{\infty}{-2} ; x)-\frac{2 m^{2}}{m^{2}+S} \mathrm{E}_{4}\left({ }_{1}^{1} ; x\right)
\end{aligned}
$$

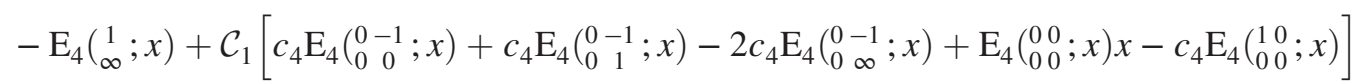

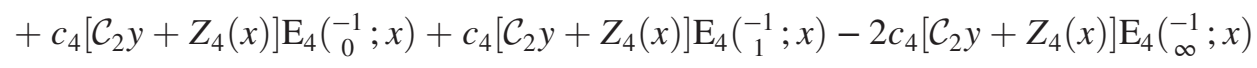

$$
\begin{aligned}
& +\left[\mathcal{C}_{2} y+Z_{4}(x)+\mathcal{C}_{1} c_{4} \log (x)\right] \mathrm{E}_{4}\left(\begin{array}{l}
0 \\
0
\end{array} ; x\right) \text {. }
\end{aligned}
$$

In the above expression we have used the coefficients $\mathcal{C}_{i}$ defined by

$$
\begin{aligned}
& \mathcal{C}_{0}=m^{2}\left(S+9 m^{2}\right) \\
& \mathcal{C}_{1}=-4 \frac{\eta_{1}}{\omega_{1}}-\frac{15 m^{4}+12 m^{2} S+S^{2}}{6\left(m^{2}+S\right)^{2} c_{4}^{2}} \\
& \mathcal{C}_{2}=-\frac{x^{3}\left(m^{2}+S\right)^{2}+x^{2}\left(3 m^{2}-S\right)\left(m^{2}+S\right)-4 m^{2} S x-2 m^{4}}{c_{4}\left[x^{4}\left(m^{2}+S\right)^{2}-2 x^{3}\left(m^{2}+S\right)^{2}+x^{2}\left(3 m^{4}+S^{2}\right)-2 m^{2} x\left(m^{2}-S\right)+m^{4}\right]}
\end{aligned}
$$

Some comments about the structure of this result are in order. First of all, note that the terms proportional to $\mathrm{E}_{4}\left(\begin{array}{l}1 \\ a_{i}\end{array} ; x\right)$ from the primitive of $\mathcal{I}_{1}$ in Eq. (4.5) exactly cancel against corresponding terms in the primitive of $\mathcal{I}_{2}$, so that the result has no logarithmic singularities at roots of the elliptic curve. Second, let us note that the term proportional to $\log x \mathrm{E}_{4}\left(\begin{array}{l}0 \\ 0\end{array} ; x\right)$ arises from the shuffle identity

$$
\mathrm{E}_{4}\left(\begin{array}{l}
0 \\
00 \\
0
\end{array} ; x\right)=\log x \mathrm{E}_{4}\left(\begin{array}{l}
0 \\
0
\end{array} ; x\right)-\mathrm{E}_{4}\left(\begin{array}{ll}
1 & 0 \\
00 & 0
\end{array}\right) .
$$

In order to obtain the final result for the second master of the sunrise, we need to evaluate the primitive at the upper boundary of the integration region at $x=1$. When we try to evaluate the primitive at $x=1$, we realize that some of the eMPLs in the result have logarithmic singularities at $x=1$, namely the eMPLs where the pole of the last integration is at $x=1$, these are

$$
\mathrm{E}_{4}\left(\begin{array}{l}
1 \\
1
\end{array} ; x\right) \quad \mathrm{E}_{4}\left(\begin{array}{c}
-1 \\
1
\end{array} ; x\right) \text { and } \mathrm{E}_{4}\left(\begin{array}{c}
-2 \\
1
\end{array} ; x\right) .
$$

The first of these eMPLs is already manifestly logarithmically divergent at $x=1$, because $\mathrm{E}_{4}\left({ }_{1}^{1} ; x\right)=\log (1-x)$. The remaining two, however, do not have the divergent logarithms manifest. In order to be able to explicitly cancel the divergent logarithms and to obtain a manifestly finite result, we need to extract the divergence from the remaining two eMPLs. The strategy for that is very simple: we start from the integral representation and subtract the pole at $x=1$. Let us illustrate this procedure on $\mathrm{E}_{4}\left(\begin{array}{c}-1 \\ 1\end{array} x\right)$. We have,

$$
\mathrm{E}_{4}\left(\frac{-1}{1} ; x\right)=\int_{0}^{x} d x^{\prime} \frac{y_{1}}{(x-1) y_{x^{\prime}}} .
$$

The integrand has a pole at $x=1$ with unit residue. We subtract the pole, and then add it back,

$\mathrm{E}_{4}\left(\begin{array}{c}-1 \\ 1\end{array} ; x\right)=\mathrm{E}_{4}\left(\begin{array}{l}1 \\ 1\end{array} x\right)+\int_{0}^{x} d x^{\prime}\left[\frac{y_{1}}{(x-1) y_{x^{\prime}}}-\frac{1}{x-1}\right]$.

The singularity is now manifest in the $\mathrm{E}_{4}\left({ }_{1}^{1} ; x\right)$ term, while the remaining term is finite. We define

$$
\varepsilon_{4}\left(\begin{array}{c}
-1 \\
1
\end{array}\right) \equiv \int_{0}^{1} d x^{\prime}\left[\frac{y_{1}}{(x-1) y_{x^{\prime}}}-\frac{1}{x-1}\right],
$$

such that

$$
\lim _{x \rightarrow 1}\left(\mathrm{E}_{4}\left(\begin{array}{c}
-1 \\
1
\end{array} ; x\right)-\mathrm{E}_{4}\left(\begin{array}{c}
1 \\
1
\end{array} ; x\right)\right)=\varepsilon_{4}\left(\begin{array}{c}
-1 \\
1
\end{array}\right) .
$$

Similarly we can regulate $\mathrm{E}_{4}\left(\frac{-2}{1} ; x\right)$ using 
$\mathrm{E}_{4}\left(\begin{array}{c}-2 \\ 1\end{array} x\right)=\mathrm{E}_{4}\left({ }_{1}^{1} ; x\right) Z_{4}(1)+\int_{0}^{x} d x^{\prime}\left[\frac{y_{1} Z_{4}(x)}{(x-1) y_{x^{\prime}}}-\frac{Z_{4}(1)}{x-1}\right]$,

and we obtain a finite limit

$$
\lim _{x \rightarrow 1}\left(\mathrm{E}_{4}\left(\begin{array}{c}
-2 \\
1
\end{array} ; x\right)-\mathrm{E}_{4}\left(\begin{array}{l}
1 \\
1
\end{array} ;\right) Z_{4}(1)\right)=\varepsilon_{4}\left(\begin{array}{c}
-2 \\
1
\end{array}\right) .
$$

Inserting the expressions for the regulated limits from Eqs. (4.29) and (4.31) into the primitive in Eq. (4.22), we see that the logarithmic divergences cancel. The final result for the second master integral reads

$$
\begin{aligned}
& \left.S_{211}\left(S, m^{2}\right)\right|_{\epsilon^{0}}=\frac{1}{\mathcal{C}_{0}}\left[-c_{4} \mathrm{E}_{4}\left(\begin{array}{c}
-2 \\
0
\end{array} ; 1\right)-c_{4} \varepsilon_{4}\left(\begin{array}{c}
-2 \\
1
\end{array}\right)+2 c_{4} \mathrm{E}_{4}\left(\begin{array}{c}
-2 \\
\infty
\end{array} ;\right)-\mathrm{E}_{4}\left(\begin{array}{c}
1 \\
\infty
\end{array} ; 1\right)\right.
\end{aligned}
$$

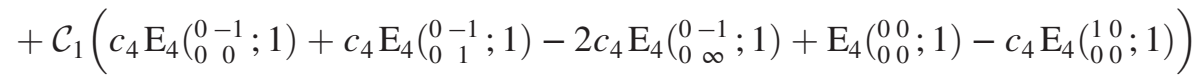

$$
\begin{aligned}
& \left.+\left(Z_{4}(1)-2 \frac{y_{1}}{c_{4}}\right)\left(c_{4} \mathrm{E}_{4}\left(\begin{array}{c}
-1 \\
0
\end{array} ; 1\right)+c_{4} \varepsilon_{4}\left(\begin{array}{c}
-1 \\
1
\end{array}\right)-2 c_{4} \mathrm{E}_{4}\left(\begin{array}{c}
-1 \\
\infty
\end{array} ; 1\right)+\mathrm{E}_{4}\left(\begin{array}{c}
0 \\
0
\end{array} 1\right)\right)\right] .
\end{aligned}
$$

While we only present the explicit result for the leading order in the $\epsilon$ expansion, it is easy to repeat the steps outlined in this section to obtain analytic results at every order in $\epsilon$ in terms of eMPLs and $Z_{4}$.

\section{A LIGHTNING SUMMARY OF ELLIPTIC POLYLOGARITHMS}

In the previous sections, we have shown that all the members of the sunrise topology can be expressed in terms of the eMPLs defined in Eqs. (3.13), (3.15) and (3.16). We discuss how to extend these results to members of the sunrise topology depending on three different masses in the next section. The goal of this section is to present a concise summary of the main concepts introduced in previous sections, and to shortly discuss some further properties of eMPLs. For a more detailed discussion of these functions and their properties, we refer to Ref. [40].

\section{A. Elliptic curves and their invariants}

Our main objects of interest are integrals whose integrands depend on a rational function of the integration variable $x$ as well as of the square root $y$ of a quartic polynomial in $x$,

$$
y=\sqrt{\left(x-a_{1}\right)\left(x-a_{2}\right)\left(x-a_{3}\right)\left(x-a_{4}\right)} .
$$

The variables $x$ and $y$ define an elliptic curve, and so we actually look at integrals on an elliptic curve. The quantity $\vec{a}=\left(a_{1}, a_{2}, a_{3}, a_{4}\right)$ is called the root vector of the elliptic curve. There are certain 'invariants' attached to an elliptic curve, the periods $\omega_{i}$ and the quasiperiods $\eta_{i}$, cf. Eqs. (4.13) and (4.15). The periods and quasiperiods are functions only of the root vector, and can be expressed in terms of the elliptic integrals of the first and second kind $\mathrm{K}$ and $\mathrm{E}$, cf. Eqs. (3.9) and (4.16). Note that a cubic polynomial also defines an elliptic curve. We do not discuss the case of a cubic polynomial in this paper, but we refer to [40].

\section{B. IBP identities}

An integral on an elliptic curve can be reduced via IBP identities to a set of basis/master integrals. The relevant IBP identities are collected in Appendix A. The kernels corresponding to the master integrals read

$$
\begin{aligned}
\psi_{0}(0, x) & =\frac{c_{4}}{y}, \\
\psi_{1}(c, x) & =\frac{1}{x-c}, \quad \psi_{-1}(c, x)=\frac{y_{c}}{y(x-c)}, \\
\psi_{1}(\infty, x) & =\frac{c_{4}}{y} Z_{4}(x), \quad \psi_{-1}(\infty, x)=\frac{x}{y}, \\
\psi_{-n}(\infty, x) & =\frac{x}{y} Z_{4}^{(n-1)}(x)-\frac{\delta_{n 2}}{c_{4}}, \\
\psi_{n}(c, x) & =\frac{1}{x-c} Z_{4}^{(n-1)}(x)-\delta_{n 2}\left(\tilde{\Phi}_{4}(x)+4 c_{4} \frac{\eta_{1}}{\omega_{1}} \frac{1}{y}\right), \\
\psi_{n}(\infty, x) & =\frac{c_{4}}{y} Z_{4}^{(n)}(x), \quad \psi_{-n}(c, x)=\frac{y_{c}}{y(x-c)} Z_{4}^{(n-1)}(x) .
\end{aligned}
$$

The quantities $Z_{4}$ and $\tilde{\Phi}_{4}$ are defined in Eqs. (4.12) and (4.9). The integration kernels are chosen such that they have at most simple poles, and so their integrated versions have at most logarithmic singularities. The quantities $Z_{4}^{(n)}$ are polynomials in $Z_{4}$ of the form

$$
Z_{4}^{(n)}(x)=\frac{(-1)^{n}}{2^{n} n !} Z_{4}(x)^{n}+\cdots
$$

with $Z_{4}^{(1)}(x)=Z_{4}(x)$, and the dots indicate terms that involve fewer powers of $Z_{4}$, such that they cancel the pole of order $n$ at $x=\infty$ of $Z_{4}(x)^{n}$. In the context of the sunrise integrals, only the case $n=1$ is relevant. We do therefore not discuss the case $n>1$ here, but we refer to the literature for a detailed discussion [40]. 


\section{Elliptic polylogarithms}

Integrating multiple times over the elliptic integration kernels in Eq. (5.2), leads to multiple elliptic polylogarithms (eMPLs)

$$
\mathrm{E}_{4}\left(\begin{array}{l}
n_{1} \ldots n_{k} \\
c_{1} \ldots c_{k}
\end{array} ; x\right)=\int_{0}^{x} d t \psi_{n_{1}}\left(c_{1}, t\right) \mathrm{E}_{4}\left(\begin{array}{c}
n_{2} \ldots n_{k} \\
c_{2} \ldots c_{k}
\end{array} ; t\right),
$$

where $n_{i} \in \mathbb{Z}, c_{i} \in \mathbb{C} \cup\{\infty\}$ and the recursion starts with $E_{4}(; x)=1 . k$ is called the length and $n_{1}+\cdots+n_{k}$ is the weight. Since the kernels have at most simple poles, eMPLs have at most logarithmic singularities.

\section{Shuffle algebra}

eMPLs form a shuffle algebra graded by the length. In other words, a product of two eMPLs can be recast in the form of a linear combination of eMPLs,

$$
\mathrm{E}_{4}\left(\vec{d}_{1} ; x\right) \mathrm{E}_{4}\left(\vec{d}_{2} ; x\right) \sum_{\vec{d} \in \vec{d}_{1} \omega \vec{d}_{2}} \mathrm{E}_{4}(\vec{d} ; x),
$$

with $\vec{d}_{1}=\left(\begin{array}{c}n_{1} \ldots n_{k} \\ c_{1} \ldots c_{k}\end{array}\right)$ and similarly for $\vec{d}_{2}$, and the sum runs over all shuffles of $\vec{d}_{1}$ and $\vec{d}_{2}$, i.e., all possible permutations of $\vec{d}_{1} \cup \vec{d}_{2}$ that preserve the relative orderings within $\vec{d}_{1}$ and $\vec{d}_{2}$.

\section{E. Completeness and independence of the integration kernels}

The integration kernels in Eq. (5.2) define a complete and independent set. In particular, eMPLs are linearly independent (for generic values of $x$ ). The set of kernels is infinite, which is a feature of the elliptic curve. In general, for every $c \in \mathbb{C} \cup\{\infty\}$ with $c \neq a_{i}$, there are two infinite towers of integration kernels $\psi_{ \pm n}(c, x), n \geq 1$. If $c=a_{i}$, then we can always reduce the tower with negative index via IBP identities [cf. Sec. IVA for an illustration of this reduction in the case of $\psi_{-1}\left(a_{i}, x\right)$, in particular Eq. (4.19)]. For the solution of a particular integral, however, only a finite number of kernels is required. Finally, every integral involving eMPLs and rational functions involving $x$ and $y$ can be performed in terms of the same class of functions. For an explicit algorithm how to perform such integrals, we refer to Appendix A and Ref. [40].

\section{F. Relationship to ordinary MPLs and elliptic integrals}

eMPLs contain various classes of special functions that are well-known in the mathematics and physics literature. In particular, Eq. (3.16) implies that ordinary MPLs are a special case of eMPLs,

$$
\mathrm{E}_{4}\left(\begin{array}{c}
1 \ldots 1 \\
c_{1} \ldots c_{k}
\end{array} ; x\right)=G\left(c_{1}, \ldots, c_{k} ; x\right), \quad c_{i} \neq \infty .
$$

In addition, the incomplete elliptic integrals of the first, second and third kinds are special cases of eMPLs. For example, if the root vector is $\vec{a}=(-1 / w,-1,1,1 / w)$, $0<w<1$, we have

$$
\begin{aligned}
\mathrm{F}\left(x \mid w^{2}\right)= & \int_{0}^{x} \frac{d t}{\sqrt{\left(1-t^{2}\right)\left(1-w^{2} t^{2}\right)}}=\frac{2}{1+w} \mathrm{E}_{4}\left({ }_{0}^{0} ; x\right), \\
\mathrm{E}\left(x \mid w^{2}\right)= & \int_{0}^{x} d t \frac{1-w^{2} t^{2}}{\sqrt{\left(1-t^{2}\right)\left(1-w^{2} t^{2}\right)}} \\
= & {\left[2(1+w) \frac{\eta_{1}}{\omega_{1}}+\frac{5-w^{2}}{3(1+w)}\right] \mathrm{E}_{4}\left(\begin{array}{l}
0 \\
0
\end{array} x\right) } \\
& -\frac{1}{2}(1+w)\left(Z_{4}(x)-Z_{4}(0)\right), \\
\Pi\left(n^{2}, x \mid w^{2}\right)= & \int_{0}^{x} \frac{d t}{\sqrt{\left(1-t^{2}\right)\left(1-w^{2} t^{2}\right)}} \frac{1}{1-n^{2} t^{2}} \\
= & \frac{1}{2 n w}\left(\frac{\mathrm{E}_{4}(-1 / n ; x)}{y_{-1 / n}}-\frac{\mathrm{E}_{4}\left(\begin{array}{l}
-1 / n \\
y_{1 / n}
\end{array} ; x\right)}{y_{1 / n}}\right) .
\end{aligned}
$$

\section{G. Relationship to the eMPLs by Brown \& Levin}

Finally, our eMPLs are closely related to multiple elliptic polylogarithms that appear in pure mathematics and string theory [36,37,43]. In particular, in Ref. [40], we show that every $\mathrm{E}_{4}$ function can be written as a linear combination of the elliptic polylogarithms of Refs. [36,37,43], and vice-versa (Up to a technical distinction which is irrelevant in the context of this paper; see Ref. [40] for details). In other words, the functions defined in Eq. (3.13) are simply an alternative basis for the eMPLs in Refs. [36,37,43]. As such, our functions deserve indeed to be called elliptic polylogarithms.

\section{SEVERAL APPLICATIONS}

In this section, we present additional applications of our elliptic polylogarithms to integrals from the sunrise topology. While in previous sections we have focused on the equal-mass case, from now on we consider the generic case with three different nonzero masses. Using IBP identities, one finds four master integrals with three propagators for the sunrise topology, which we may choose as

$$
S_{111}, \quad S_{211}, \quad S_{121} \text { and } S_{112} .
$$

The three master integrals with an additional power on one of the propagators are obviously related by a simple permutation of the masses $m_{i}$. It will therefore be sufficient to discuss the integrals $S_{111}$ and $S_{211}$, and the remaining integrals can be obtained by symmetry. For this reason, we will again refer to $S_{111}$ and $S_{211}$ as the first and second master integrals of the sunrise topology.

Analytic results for the first master integral $S_{111}$ with three different masses can be found in Refs. [26,27], while one of the main results of this paper is to present an analytic expression for $S_{211}$ with different masses for the first time. In order to calculate this result, we take an approach that is complementary to the Feynman parameters used in the 
equal-mass case: we compute analytic expressions for $S_{111}$ and $S_{211}$ from dispersion relations. In this way, we demonstrate the flexibility of our language of elliptic polylogarithms, and at the same time we obtain more compact representations for the sunrise integrals in the case of three different masses [44].

To compute the sunrise integrals in the dispersive approach, we begin by first computing the imaginary part of the master integrals. The imaginary part can be computed using the optical theorem by replacing all propagators of the sunrise diagram by on-shell $\delta$ functions, and we discuss in detail the computation of the maximal cut of $S_{111}$ in terms of eMPLs. Once the imaginary part is obtained, we can recover the master integrals $S_{111}$ and $S_{211}$ by performing a dispersion integral.

\section{A. The maximal cut of the first master integral}

In this section, we discuss the computation of the maximal cut of $S_{111}$. In Ref. [29], it was shown that the imaginary part of $S_{111}$ can be written as follows

$$
\begin{aligned}
\operatorname{Im} & S_{111}\left(s, m_{1}^{2}, m_{2}^{2}, m_{3}^{2}\right) \\
& =S_{\epsilon} \int_{\left(m_{2}+m_{3}\right)^{2}}^{\left(\sqrt{s}-m_{1}\right)^{2}} d x \frac{\left(\frac{R_{2}\left(x, m_{2}^{2}, m_{3}^{2}\right) R_{2}\left(s, x, m_{1}^{2}\right)}{s x}\right)^{(d-2) / 2}}{\sqrt{R_{2}\left(x, m_{2}^{2}, m_{3}^{2}\right) R_{2}\left(s, x, m_{1}^{2}\right)}},
\end{aligned}
$$

with $s=-S=p^{2}$ and where we defined

$$
S_{\epsilon}=\frac{2^{d} \pi^{3 / 2}}{\Gamma\left(3-\frac{d}{2}\right) \Gamma\left(\frac{d-1}{2}\right)} \quad \text { with } \quad \lim _{d \rightarrow 2} S_{\epsilon} \rightarrow 4 \pi,
$$

and the Källen function is

$$
R_{2}(x, y, z)=x^{2}+y^{2}+z^{2}-2 x y-2 x z-2 y z .
$$

Since the Källen function is a polynomial of degree two, we can immediately recognize the square root of a quartic polynomial in the denominator of Eq. (6.2). The quartic polynomial is given by

$$
\begin{aligned}
y^{2} & \equiv R_{2}\left(x, m_{2}^{2}, m_{3}^{2}\right) R_{2}\left(s, x, m_{1}^{2}\right) \\
& =\left(x-b_{1}\right)\left(x-b_{2}\right)\left(x-b_{3}\right)\left(x-b_{4}\right),
\end{aligned}
$$

where the roots are given by

$b_{1}=\left(m_{2}-m_{3}\right)^{2}, \quad b_{2}=\left(m_{2}+m_{3}\right)^{2}$,

$b_{3}=\left(m_{1}-\sqrt{s}\right)^{2} \quad$ and $\quad b_{4}=\left(m_{1}+\sqrt{s}\right)^{2}$.

For $s>\left(m_{1}+m_{2}+m_{3}\right)^{2}$, the roots are real and ordered as $b_{1}<b_{2}<b_{3}<b_{4}$, which we assume in the following. We also assume that the three masses $m_{i}$ are distinct and nonvanishing, so that no two roots coincide.

We can now rewrite Eq. (6.2) manifestly as integral on an elliptic curve,

$$
\begin{aligned}
& \operatorname{Im} S_{111}\left(s, m_{1}^{2}, m_{2}^{2}, m_{3}^{2}\right) \\
& \quad=S_{\epsilon} \int_{b_{2}}^{b_{3}} \frac{d x}{y}\left(\frac{R_{2}\left(x, m_{2}^{2}, m_{3}^{2}\right) R_{2}\left(s, x, m_{1}^{2}\right)}{s x}\right)^{(d-2) / 2} \\
& =S_{\epsilon}\left(C_{111}^{(0)}\left(s, m_{1}^{2}, m_{2}^{2}, m_{3}^{2}\right)+\epsilon C_{111}^{(1)}\left(s, m_{1}^{2}, m_{2}^{2}, m_{3}^{2}\right)+\mathcal{O}\left(\epsilon^{2}\right)\right) .
\end{aligned}
$$

The leading term in $\epsilon$ is given by

$$
\begin{aligned}
C_{111}^{(0)}\left(s, m_{1}^{2}, m_{2}^{2}, m_{3}^{2}\right) & =\int_{b_{2}}^{b_{3}} \frac{d x}{y}=\int_{b_{2}}^{b_{3}} d x \frac{\psi_{0}(0, x)}{c_{4}} \\
& =\frac{1}{c_{4}}\left[\mathrm{E}_{4}\left(\begin{array}{l}
0 \\
0
\end{array} ; b_{3}\right)-\mathrm{E}_{4}\left(\begin{array}{l}
0 \\
0
\end{array} ; b_{2}\right)\right],
\end{aligned}
$$

where in the second step we have recognized the integration kernel $\psi_{0}(0, x)$. The coefficient $C_{111}^{(1)}$ can be represented by the integral

$$
\begin{aligned}
& C_{111}^{(1)}\left(s, m_{1}^{2}, m_{2}^{2}, m_{3}^{2}\right)=\int_{b_{2}}^{b_{3}} \frac{d x}{y}\left[\log \left(R_{2}\left(x, m_{2}^{2}, m_{3}^{2}\right) R_{2}\left(s, x, m_{1}^{2}\right)\right)-\log s-\log x\right] \\
& =\int_{b_{2}}^{b_{3}} \frac{d x}{y}\left[-\log s-\log x+\log \left(b_{1} b_{2} b_{3} b_{4}\right)+\sum_{i=1}^{4} \log \left(1-\frac{x}{b_{i}}\right)\right]
\end{aligned}
$$

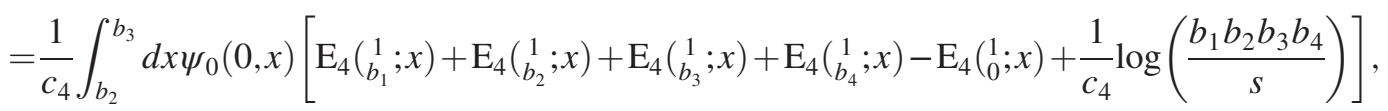

where in the last step we have used the fact that ordinary MPLs are a subset of eMPLs, cf. Eq. (5.6). The remaining integral can now be performed by using the recursive definition of eMPLs in Eq. (3.13), with the result

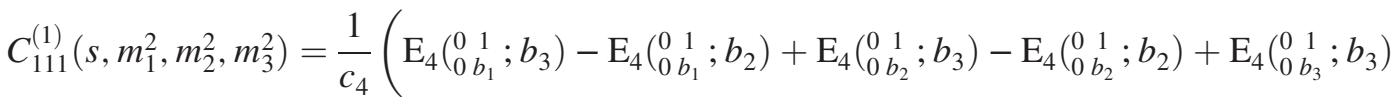

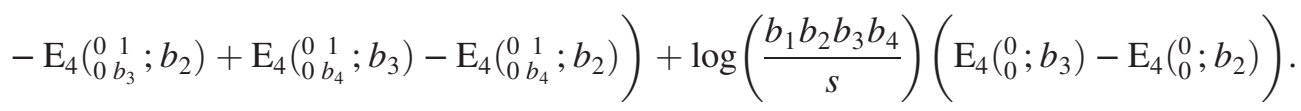


Let us conclude this section by commenting on how the results of this section generalize to higher orders in $\epsilon$. From Eq. (6.7), we see that the integrand at any order contains products of logarithms only. Using the shuffle algebra properties of MPLs and eMPLs, and the fact that ordinary MPLs are a subset of eMPLs, we can see that the integrand can always be written in terms of $\mathrm{E}_{4}$ functions. The resulting integrals can then always easily be performed in terms of eMPLs, proving that the maximal cut of the sunrise integral can be expressed in terms of eMPLs to all orders in dimensional regularization.

\section{B. The first master integral from a dispersion relation}

In this section, we present the computation of the master integrals $S_{111}$ and $S_{211}$ using a dispersive approach. As we will see, this allows us to obtain a very compact representation for all master integrals, even in the unequal mass case.

Let us go back to the formula for the imaginary part of the two-loop massive sunrise with different masses and for general values of the dimensions $d$, Eq. (6.2). We can reconstruct the full sunrise integral through a dispersion relation as follows

$$
\begin{aligned}
S_{111} & \left(s, m_{1}^{2}, m_{2}^{2}, m_{3}^{2}\right) \\
= & \frac{1}{\pi} \int_{m_{123}^{2}}^{\infty} \frac{d u}{u-s} \operatorname{Im} S_{111}\left(u, m_{1}^{2}, m_{2}^{2}, m_{3}^{2}\right) \\
= & \frac{S_{\epsilon}}{\pi} \int_{m_{123}^{2}}^{\infty} \frac{d u}{u-s} \int_{\left(m_{2}+m_{3}\right)^{2}}^{\left(\sqrt{s}-m_{1}\right)^{2}} \frac{d t}{\sqrt{R_{2}\left(t, m_{2}^{2}, m_{3}^{2}\right) R_{2}\left(u, t, m_{1}^{2}\right)}} \\
& \times\left(\frac{R_{2}\left(t, m_{2}^{2}, m_{3}^{2}\right) R_{2}\left(u, t, m_{1}^{2}\right)}{u t}\right)^{(d-2) / 2}
\end{aligned}
$$

where $S_{\epsilon}$ was defined in Eq. (6.3), $m_{123}^{2}=\left(m_{1}+m_{2}+m_{3}\right)^{2}$ and we omitted the small imaginary part associated to $s$, $s \rightarrow s+i 0^{+}$. As a first step, we exchange the integrals and write

$$
\begin{aligned}
& S_{111}\left(s, m_{1}^{2}, m_{2}^{2}, m_{3}^{2}\right) \\
& =\frac{S_{\epsilon}}{\pi} \int_{\left(m_{2}+m_{3}\right)^{2}}^{\infty} \frac{d t}{\sqrt{R_{2}\left(t, m_{2}^{2}, m_{3}^{2}\right)}}\left(\frac{R_{2}\left(t, m_{2}^{2}, m_{3}^{2}\right)}{t}\right)^{(d-2) / 2} \\
& \quad \times \int_{\left(\sqrt{t}+m_{1}\right)^{2}}^{\infty} \frac{d u}{u-s} \frac{1}{\sqrt{R_{2}\left(u, t, m_{1}^{2}\right)}}\left(\frac{R_{2}\left(u, t, m_{1}^{2}\right)}{u}\right)^{(d-2) / 2} .
\end{aligned}
$$

Upon inspecting Eq. (6.12), since the integral over $u$ contains only one quadratic square root, it is obvious that this integral can easily be performed in terms of multiple polylogarithms at every order in $\epsilon$. The result can then be integrated over $t$ in terms of our $E_{4}$ functions. For simplicity, we limit ourselves to the leading order in $\epsilon$, though we stress that there is no conceptual obstacle to extend the result to higher orders. Furthermore, we assume without loss of generality that the masses are ordered according to $m_{3}<m_{2}<m_{1}$, and we work below threshold to deal with real quantities only, i.e., $0<s<m_{123}^{2}$. We find

$$
\begin{aligned}
& S_{111}\left(s, m_{1}^{2}, m_{2}^{2}, m_{3}^{2}\right) \\
& =4 \int_{\left(m_{2}+m_{3}\right)^{2}}^{\infty} \frac{d t}{\sqrt{R_{2}\left(t, m_{2}^{2}, m_{3}^{2}\right)}} \\
& \quad \times \int_{\left(\sqrt{t}+m_{1}\right)^{2}}^{\infty} \frac{d u}{u-s} \frac{1}{\sqrt{R_{2}\left(u, t, m_{1}^{2}\right)}} .
\end{aligned}
$$

We can now perform explicitly the integral over $u$ and we find

$$
\begin{aligned}
S_{111}\left(s, m_{1}^{2}, m_{2}^{2}, m_{3}^{2}\right)= & 2 \int_{\left(m_{2}+m_{3}\right)^{2}}^{\infty} \frac{d t}{\sqrt{R_{2}\left(t, m_{2}^{2}, m_{3}^{2}\right) R_{2}\left(s, t, m_{1}^{2}\right)}} \\
& \times \ln \left(\frac{t+m_{1}^{2}-s+\sqrt{R_{2}\left(s, t, m_{1}^{2}\right)}}{t+m_{1}^{2}-s-\sqrt{R_{2}\left(s, t, m_{1}^{2}\right)}}\right) .
\end{aligned}
$$

Finally, we change of variables according to

$$
t=b_{3}+\frac{\left(b_{4}-b_{3}\right)}{4} \frac{(1+x)^{2}}{x},
$$

where the four roots $b_{1}, b_{2}, b_{3}, b_{4}$ are defined in Eq. (6.6), and we are left with

$$
\begin{aligned}
& S_{111}\left(s, m_{1}^{2}, m_{2}^{2}, m_{3}^{2}\right) \\
& =\frac{2}{\sqrt{s} m_{1}} \int_{0}^{Q_{2}} \frac{d x}{\sqrt{\left(x-Q_{1}\right)\left(x-1 / Q_{1}\right)\left(x-Q_{2}\right)\left(x-1 / Q_{2}\right)}} \\
& \quad \times \ln \left(\frac{q+x}{x(1+x q)}\right),
\end{aligned}
$$

where we defined

$$
\begin{aligned}
q & =\frac{\sqrt{s}}{m_{1}}, \quad Q_{1}=\frac{\sqrt{a_{1}-a_{3}}-\sqrt{a_{1}-a_{4}}}{\sqrt{a_{1}-a_{3}}+\sqrt{a_{1}-a_{4}}}, \\
Q_{2} & =\frac{\sqrt{a_{2}-a_{3}}-\sqrt{a_{2}-a_{4}}}{\sqrt{a_{2}-a_{3}}+\sqrt{a_{2}-a_{4}}} .
\end{aligned}
$$

It is now entirely straightforward to integrate Eq. (6.16) in terms of our $E_{4}$ functions. We recast the logarithms in the form of eMPLs functions, and we obtain

$$
\begin{aligned}
\ln \left(\frac{q+x}{x(1+x q)}\right)= & \ln q+\ln (1+x / q)-\ln x-\ln (1+x q) \\
= & \ln q+\mathrm{E}_{4}\left(\begin{array}{c}
1 \\
-q
\end{array} ; x\right)-\mathrm{E}_{4}\left({ }_{0}^{1} ; x\right) \\
& -\mathrm{E}_{4}\left(\begin{array}{c}
1 \\
-1 / q
\end{array} ; x\right)
\end{aligned}
$$


which immediately yields

$$
S_{111}\left(s, m_{1}^{2}, m_{2}^{2}, m_{3}^{2}\right)=\frac{4 \sqrt{2}}{\sqrt{P_{0}}} F_{1}^{(1)}\left(s, m_{1}^{2}, m_{2}^{2}, m_{3}^{2}\right)
$$

with the definitions

$$
\begin{aligned}
P_{0}= & 2 m_{1}^{2} m_{2}^{2}+2 m_{1}^{2} m_{3}^{2}+2 m_{2}^{2} m_{3}^{2}+2\left(m_{1}^{2}+m_{2}^{2}+m_{3}^{2}\right) s \\
& -\left(m_{1}^{4}+m_{2}^{4}+m_{3}^{4}\right)-s^{2} \\
& -\sqrt{a_{1}-a_{3}} \sqrt{a_{1}-a_{4}} \sqrt{a_{2}-a_{3}} \sqrt{a_{2}-a_{4}},
\end{aligned}
$$

and

$$
\begin{aligned}
F_{1}^{(1)}\left(s, m_{1}^{2}, m_{2}^{2}, m_{3}^{2}\right)= & \mathrm{E}_{4}\left(\begin{array}{c}
01 \\
00
\end{array} ; Q_{2}\right)+\mathrm{E}_{4}\left(\begin{array}{c}
0 \\
0-1 / q
\end{array} ; Q_{2}\right) \\
& -\mathrm{E}_{4}\left(\begin{array}{cc}
0 & 1 \\
0-q & ;
\end{array} Q_{2}\right)-\ln q \mathrm{E}_{4}\left(\begin{array}{l}
0 \\
0
\end{array} ; Q_{2}\right) .
\end{aligned}
$$

\section{The second master integral from a dispersion relation}

In this section, we extend the results of the previous section to the second master integral of the sunrise integrals with different masses. Without loss of generality, we discuss $S_{121}$, and the other cases are obtained by symmetry. In addition, the second master integral is related to the first master integral by differentiation,

$S_{121}\left(s, m_{1}^{2}, m_{2}^{2}, m_{3}^{2}\right)=\frac{\partial}{\partial m_{2}^{2}} S_{111}\left(s, m_{1}^{2}, m_{2}^{2}, m_{3}^{2}\right)$.

Hence, we can obtain an integral representation of $S_{121}$ by simply differentiating with respect to the mass in the dispersive representation of $S_{111}$ in Eq. (6.16). However, upon differentiating under the integral sign, we generate a potential end-point singularity in the upper integration limit $x \rightarrow Q_{2}$, which we need to regularize. We write

$$
\begin{aligned}
S_{121}\left(s, m_{1}^{2}, m_{2}^{2}, m_{3}^{2}\right)= & \frac{\partial}{\partial m_{2}^{2}} S_{111}\left(s, m_{1}^{2}, m_{2}^{2}, m_{3}^{2}\right) \\
= & \frac{2}{\sqrt{s} m_{1}} \int_{0}^{Q_{2}-\delta} \frac{d x}{\sqrt{\left(x-Q_{1}\right)\left(x-1 / Q_{1}\right)\left(x-Q_{2}\right)\left(x-1 / Q_{2}\right)}} \ln \left(\frac{q+x}{x(1+x q)}\right) \\
& \times \frac{x\left(m_{1} \sqrt{s}+\left(m_{1}^{2}-m_{2}^{2}+m_{3}^{2}+s\right) x+m_{1} \sqrt{s} x^{2}\right)}{m_{1}^{2} s\left(x-Q_{1}\right)\left(x-1 / Q_{1}\right)\left(x-Q_{2}\right)\left(x-1 / Q_{2}\right)} \\
& +\frac{2}{\sqrt{s} m_{1}} \frac{1}{\sqrt{-\delta}} \frac{1}{\sqrt{\left(Q_{2}-Q_{1}\right)\left(Q_{2}-1 / Q_{1}\right)\left(Q_{2}-1 / Q_{2}\right)}} \ln \left(\frac{q+Q_{2}}{Q_{2}\left(1+Q_{2} q\right)}\right),
\end{aligned}
$$

where we introduced a small regularization parameter $\delta$. If we perform partial fractioning in $x$ and use the expression of the logarithms in terms of $E_{4}$ functions, we immediately see that we are left with the following types of integrals to do

$$
\int_{0}^{Q_{2}-\delta} \frac{d x}{y} \frac{1}{x-Q} \mathrm{E}_{4}\left(\begin{array}{l}
n \\
c
\end{array} ; x\right), \quad Q \in\left\{1 / Q_{1}, Q_{1}, Q_{2}, 1 / Q_{2}\right\},
$$

for different values of $(n, c)$. The integrals above can be performed in a straightforward way in terms of $E_{4}$ functions using the techniques introduced in previous sections. In the final result, all poles in $\delta$ cancel, and we can safely take the limit $\delta \rightarrow 0$. We find that $S_{121}\left(s, m_{1}^{2}, m_{2}^{2}, m_{3}^{2}\right)$ can be written in a very compact form as follows

$$
\begin{aligned}
S_{121}\left(s, m_{1}^{2}, m_{2}^{2}, m_{3}^{2}\right)= & \frac{1}{D \sqrt{2 P_{0}}}\left[F_{2}^{(-1)}\left(s, m_{1}^{2}, m_{2}^{2}, m_{3}^{2}\right)-\frac{4}{3} P_{3} F_{1}^{(1)}\left(s, m_{1}^{2}, m_{2}^{2}, m_{3}^{2}\right)\right] \\
& +\frac{\sqrt{2 P_{0}}}{D}\left[F_{2}^{(1)}\left(s, m_{1}^{2}, m_{2}^{2}, m_{3}^{2}\right)-2 \frac{\eta_{1}}{\omega_{1}} P_{1} S\left(s, m_{1}^{2}, m_{2}^{2}, m_{3}^{2}\right)\right]+\frac{1}{D} F_{2}^{(0)}\left(s, m_{1}^{2}, m_{2}^{2}, m_{3}^{2}\right)
\end{aligned}
$$

where we introduced the further abbreviations

$$
\begin{aligned}
\mu_{1} & =-m_{1}+m_{2}+m_{3}, \quad \mu_{2}=m_{1}-m_{2}+m_{3}, \quad \mu_{3}=m_{1}+m_{2}-m_{3}, \\
D & =m_{2}^{2}\left(s-m_{123}^{2}\right)\left(s-\mu_{1}^{2}\right)\left(s-\mu_{2}^{2}\right)\left(s-\mu_{3}^{2}\right),
\end{aligned}
$$

and

$$
F_{2}^{(-1)}\left(s, m_{1}^{2}, m_{2}^{2}, m_{3}^{2}\right)=-\frac{4}{3} P_{2} \mathrm{E}_{4}\left({ }_{0}^{0} ; Q_{2}\right)
$$




$$
\begin{aligned}
F_{2}^{(0)}\left(s, m_{1}^{2}, m_{2}^{2}, m_{3}^{2}\right)=- & P_{4} \mathrm{E}_{4}\left({ }_{\infty}^{-1} ; Q_{2}\right)+P_{5} \mathrm{E}_{4}\left({ }_{-1 / q}^{-1} ; Q_{2}\right)-P_{6} \mathrm{E}_{4}\left({ }_{-q}^{-1} ; Q_{2}\right)+P_{7} \mathrm{E}_{4}\left({ }_{0}^{-1} ; Q_{2}\right)-\ln \frac{q}{Q_{2}} \\
F_{2}^{(1)}\left(s, m_{1}^{2}, m_{2}^{2}, m_{3}^{2}\right)= & P_{1}\left[-\frac{1}{2} \mathrm{E}_{4}\left({ }_{0}^{2} ; Q_{2}\right)-\frac{1}{2} \mathrm{E}_{4}\left({ }_{-1 / q}^{2} ; Q_{2}\right)+\frac{1}{2} \mathrm{E}_{4}\left(_{-q}^{2} ; Q_{2}\right)+\frac{2 \eta_{1}}{\omega_{1}} \mathrm{E}_{4}\left({ }_{0}^{0} ; Q_{2}\right)\right. \\
& \left.-\frac{i \pi}{\omega_{1}} \ln \left(\frac{\left(q+Q_{2}\right)}{Q_{2}\left(1+q Q_{2}\right)}\right)+\frac{1}{2} \ln \left(\frac{q}{Q_{2}}\right) Z_{4}^{(1)}(0)\right]
\end{aligned}
$$

The polynomials $P_{i}$ appearing in the previous expressions are given by

$$
\begin{aligned}
& P_{1}=m_{1}^{4}+2 m_{1}^{2} m_{2}^{2}-2 m_{1}^{2} m_{3}^{2}-3 m_{2}^{4}+2 m_{2}^{2} m_{3}^{2}+m_{3}^{4}+s^{2}-2 s\left(m_{1}^{2}-m_{2}^{2}+m_{3}^{2}\right), \\
& P_{2}=m_{1}^{8}+3 m_{1}^{6} m_{2}^{2}-7 m_{1}^{6} m_{3}^{2}-27 m_{1}^{4} m_{2}^{4}+18 m_{1}^{4} m_{2}^{2} m_{3}^{2}+9 m_{1}^{4} m_{3}^{4}+17 m_{1}^{2} m_{2}^{6}+29 m_{1}^{2} m_{2}^{4} m_{3}^{2} \\
& -45 m_{1}^{2} m_{2}^{2} m_{3}^{4}-m_{1}^{2} m_{3}^{6}+6 m_{2}^{8}-16 m_{2}^{6} m_{3}^{2}+12 m_{2}^{4} m_{3}^{4}-2 m_{3}^{8}-5 s^{4} \\
& +s^{3}\left(2 m_{1}^{2}-3 m_{2}^{2}+23 m_{3}^{2}\right)+s^{2}\left(12 m_{1}^{4}-39 m_{1}^{2} m_{2}^{2}-5 m_{1}^{2} m_{3}^{2}+51 m_{2}^{4}-18 m_{2}^{2} m_{3}^{2}-33 m_{3}^{4}\right) \\
& +s\left(-10 m_{1}^{6}-9 m_{1}^{4} m_{2}^{2}+37 m_{1}^{4} m_{3}^{2}+44 m_{1}^{2} m_{2}^{4}-24 m_{1}^{2} m_{2}^{2} m_{3}^{2}-20 m_{1}^{2} m_{3}^{4}-49 m_{2}^{6}-13 m_{2}^{4} m_{3}^{2}+45 m_{2}^{2} m_{3}^{4}+17 m_{3}^{6}\right), \\
& P_{3}=-m_{123} \mu_{1} \mu_{2} \mu_{3}\left(m_{1}^{4}-4 m_{1}^{2} m_{2}^{2}-2 m_{1}^{2} m_{3}^{2}+3 m_{2}^{4}-4 m_{2}^{2} m_{3}^{2}+m_{3}^{4}\right) \\
& +s^{4}-2 s^{3}\left(2 m_{1}^{2}+3 m_{2}^{2}+2 m_{3}^{2}\right)+2 s^{2}\left(3 m_{1}^{4}+3 m_{1}^{2} m_{2}^{2}+2 m_{1}^{2} m_{3}^{2}+6 m_{2}^{4}+3 m_{2}^{2} m_{3}^{2}+3 m_{3}^{4}\right) \\
& -2 s\left(2 m_{1}^{6}-3 m_{1}^{4} m_{2}^{2}-2 m_{1}^{4} m_{3}^{2}-4 m_{1}^{2} m_{2}^{4}+30 m_{1}^{2} m_{2}^{2} m_{3}^{2}-2 m_{1}^{2} m_{3}^{4}+5 m_{2}^{6}-4 m_{2}^{4} m_{3}^{2}-3 m_{2}^{2} m_{3}^{4}+2 m_{3}^{6}\right) \text {, } \\
& P_{4}=m_{1}^{6}+m_{1}^{4} m_{2}^{2}-m_{1}^{4} m_{3}^{2}+3 m_{1}^{2} m_{2}^{4}-2 m_{1}^{2} m_{2}^{2} m_{3}^{2}-m_{1}^{2} m_{3}^{4}-5 m_{2}^{6}-5 m_{2}^{4} m_{3}^{2}+9 m_{2}^{2} m_{3}^{4}+m_{3}^{6} \\
& -3 s^{3}+7 s^{2}\left(m_{1}^{2}-m_{2}^{2}+m_{3}^{2}\right)+s\left(-5 m_{1}^{4}-2 m_{1}^{2} m_{2}^{2}+2 m_{1}^{2} m_{3}^{2}+15 m_{2}^{4}-10 m_{2}^{2} m_{3}^{2}-5 m_{3}^{4}\right), \\
& P_{5}=m_{1}^{6}-7 m_{1}^{4} m_{2}^{2}-m_{1}^{4} m_{3}^{2}+3 m_{1}^{2} m_{2}^{4}+14 m_{1}^{2} m_{2}^{2} m_{3}^{2}-m_{1}^{2} m_{3}^{4}+3 m_{2}^{6}-5 m_{2}^{4} m_{3}^{2}+m_{2}^{2} m_{3}^{4}+m_{3}^{6} \\
& -s^{3}+s^{2}\left(3 m_{1}^{2}+5 m_{2}^{2}+3 m_{3}^{2}\right)+s\left(-3 m_{1}^{4}+2 m_{1}^{2} m_{2}^{2}-2 m_{1}^{2} m_{3}^{2}-7 m_{2}^{4}-6 m_{2}^{2} m_{3}^{2}-3 m_{3}^{4}\right), \\
& P_{6}=-m_{123} \mu_{1} \mu_{2} \mu_{3}\left(m_{1}^{2}-3 m_{2}^{2}-m_{3}^{2}\right)-s^{3}+s^{2}\left(3 m_{1}^{2}+7 m_{2}^{2}+m_{3}^{2}\right) \\
& +s\left(-3 m_{1}^{4}-2 m_{1}^{2} m_{2}^{2}+2 m_{1}^{2} m_{3}^{2}-3 m_{2}^{4}-14 m_{2}^{2} m_{3}^{2}+m_{3}^{4}\right), \\
& P_{7}=-m_{123} \mu_{1} \mu_{2} \mu_{3}\left(m_{1}^{2}+m_{2}^{2}-m_{3}^{2}\right)+s^{3}+s^{2}\left(-m_{1}^{2}-m_{2}^{2}-3 m_{3}^{2}\right) \\
& +s\left(-m_{1}^{4}+10 m_{1}^{2} m_{2}^{2}-2 m_{1}^{2} m_{3}^{2}-m_{2}^{4}-2 m_{2}^{2} m_{3}^{2}+3 m_{3}^{4}\right) \text {. }
\end{aligned}
$$

Finally, we have checked all our results, both from the Feynman Parameters representation and from the dispersion relation numerically against $\mathrm{SecDec} 3$ [45] and pySecDec [46] and found perfect agreement for different values of the kinematical invariants.

\section{CONCLUSION}

In this paper, we have presented an algorithm for computing Feynman integrals which involve square roots of quartic polynomials in terms of iterated integrals on the corresponding elliptic curves. We showed how these iterated integrals can be reduced to a basis of elliptic integral kernels using integration-by-parts identities. Since our kernels have only simple poles, they define a class of functions on the elliptic curve which deserve to be called elliptic multiple polylogarithms. The mathematical details behind the construction of these functions are spelled out in a companion paper [40]. Here, instead, we have focussed on demonstrating their use to solve an important problem in high energy physics which has received a lot of attention in the last decade, namely the computation of the master integrals of the massive twoloop sunrise graph. To demonstrate the flexibility of our approach, we studied the complete set of master integrals of the two-loop sunrise graph in the equal and different mass case, both by direct integration over the Feynman parameters and using a dispersion relation. To the best of our knowledge, this is the first time that the complete set of master integrals of the unequal mass two-loop sunrise graph are computed explicitly in terms of elliptic polylogarithms.

Let us make a comment how some of the results of this paper are connected to other results in the literature. Very recently, a paper came out [12], which has addressed the calculation of similar integrals to the ones considered here in terms of ad-hoc elliptic generalizations of Goncharov polylogarithms. In contrast to Ref. [12], we believe that our 
approach is more general as it is based on a mathematically well-defined class of functions, closed under taking primitives, which can be proved to be substantially equivalent to the multiple elliptic polylogarithms defined in the mathematical literature [34-36]. We recall here that a central result of Ref. [36] consisted in proving that this class of functions is sufficient to integrate any rational function on a given elliptic curve.

The mathematical details of the construction of our set of functions, together with the proof of their equivalence to the functions defined in Ref. [36], are provided in a companion paper, Ref. [40], where also many other nontrivial examples are worked out in detail. Given the generality of our approach, we expect that our definition of elliptic polylogarithms will be applicable to a wide range of problems: from phenomenological calculations of massive Feynman integrals in the Standard Model, to calculations of amplitudes in $\mathcal{N}=4$ super Yang-Mills and string theory. In particular, it will be interesting to examine the recent representation [17] of the famous ten-point double box in $\mathcal{N}=4$ to see if it can be integrated in terms of our functions.

As a last remark, one very important aspect that we plan to study in the near future is the use of our functions in the context of the differential equations method [47-50], which has proven to be extremely powerful to compute complicated multiloop Feynman integrals in terms of multiple polylogarithms. In general, we expect the differential equations with respect to the kinematic invariants to translate in our formalism into derivatives of our functions with respect to the branch points that define the elliptic curve. A first example of this has been worked out for a special subclass of these functions appearing in the computation of the imaginary part of the equal mass two-loop sunrise graph in Ref. [31]. A proper understanding of these derivatives in the general case will be essential not only to extend the use of our functions to more complicated problems, but also to properly define a symbol calculus for elliptic polylogarithms in a similar way as it was done for Goncharov polylogarithms [6,7].

\section{ACKNOWLEDGMENTS}

The authors are grateful to Nils Matthes, Erik Panzer, Brenda Penante and Ettore Remiddi for stimulating discussions, and to the ETH Zürich and the Pauli Center for Theoretical Studies for the organization of the workshop, "The elliptic/missing Feynman integrals," where some of the ideas presented in this paper were first discussed. This research was supported by the ERC Grant No. 637019, "MathAm," the U.S. Department of Energy (DOE) under Contract No. DE-AC02-76SF00515 and the Munich Institute for Astro- and Particle Physics (MIAPP) of the DFG cluster of excellence, Origin and Structure of the Universe, funding for workshop at the MIAPP (Munich institute for Astro- and Particle Physics).

\section{APPENDIX A: INTEGRATION-BY-PARTS IDENTITIES FOR EMPLS}

In this appendix, we will discuss the IBP identities allowing to express a general integral on the elliptic curve in terms of integrals over the integration kernels provided in Eq. (5.2). The most general integral on the elliptic curve has an integrand of the form

$$
I(x, y)=R(x, y) \mathcal{X}(x),
$$

where $R(x, y)$ is a rational function, and $\mathcal{X}(x)=$ $Z_{4}(x){ }^{\alpha} \mathrm{E}_{4}\left(\frac{\vec{n}}{\vec{c}} ; x\right)$ with $\alpha$ a positive integer. The integer $\alpha+|\vec{n}|$ is called the total length of $\mathcal{X}$.

The strategy is as follows: in a first step we can perform partial fractioning in $x$ to write the rational function $R(x, y)$ in terms of members of four families of integrals. While those families are independent with respect to partial fractioning, their members are related through IBP identities. Correspondingly, in a second step, a set of master integrals must be identified within each family.

The four families suitable for the integrand in Eq. (A1) read

$$
\begin{aligned}
A_{k}[\mathcal{X}] & =\int d x x^{k} \mathcal{X}(x), & B_{c, k}[\mathcal{X}] & =\int \frac{d x}{(x-c)^{k}} \mathcal{X}(x), \\
C_{k}[\mathcal{X}] & =\int \frac{d x}{y} x^{k} \mathcal{X}(x), & D_{c, k}[\mathcal{X}] & =\int \frac{d x}{y(x-c)^{k}} \mathcal{X}(x),
\end{aligned}
$$

where $k$ is an integer.

The identification of the set of master integrals is done by recognizing identities recursive in the variable $k$ whose recursion terminates for a certain value of the recursion parameter. Let us show this on the example of the family $A_{k}[\mathcal{X}]$ : they satisfy the following relation

$$
A_{k}[\mathcal{X}]=\frac{x^{k+1}}{k+1} \mathcal{X}(x)-\frac{1}{k+1} A_{k+1}\left[\partial_{x} \mathcal{X}\right] .
$$

Each time this relation is used, the value of $k$ is increased, while the total length $\ell$ of the integrand $\mathcal{X}$ in the second term is lowered through the derivative. Reaching $\ell=0$, the integral is elementary. However, before getting there the recursion could reach the value $k=-1$ : this integral cannot be reduced further and is thus the master integral for the family $A_{k}[\mathcal{X}]$.

For the other families, similar but algebraically more involved recursion relations exist. Here we only summarize some of the features of these recursions, and we refer to Ref. [40] for a detailed treatment. First, the recursion for the $C$-family has depth four, leading to more than one master integral. Second, one will have to pay attention to the case where a shift $c$ in the integration kernels of family $D$ is a zero of the quartic polynomial. Finally, one can identify the following set of master integrals: 


$$
\begin{array}{rlrl}
A_{-1}[\mathcal{X}] & =\int \frac{d x}{x} \mathcal{X}(x), & B_{c, 1}[\mathcal{X}]=\int \frac{d x}{x-c} \mathcal{X}(x), \\
C_{-1}[\mathcal{X}] & =\int \frac{d x}{y x} \mathcal{X}(x), & C_{0}[\mathcal{X}]=\int \frac{d x}{y} \mathcal{X}(x), \\
C_{1}[\mathcal{X}] & =\int \frac{x d x}{y} \mathcal{X}(x), & C_{2}[\mathcal{X}]=\int \frac{x^{2} d x}{y} \mathcal{X}(x), \\
D_{c, 1}[\mathcal{X}] & =\int \frac{d x}{y(x-c)} \mathcal{X}(x) .
\end{array}
$$

These integrals are in one-to-one-correspondence with the integration kernels defined in Eq. (5.2): the associated integrals can then be easily done employing Eq. (3.13).

Finally, we mention that there is one potentially problematic case: $C_{2}\left[Z_{4}(x)^{n} \mathrm{E}_{4}\right]$. This integral requires the use of special identities to rewrite powers of the function $Z_{4}$. Higher powers of $Z_{4}$ are, however, not important for the examples discussed in this paper. We therefore refer the reader to Ref. [40] for a detailed discussion of this case.

\section{APPENDIX B: INTEGRALS APPEARING IN THE SECOND MASTER}

Here we list the integrals contributing to the primitive of $\mathcal{I}_{2}$ defined in Eq. (4.4). These integrals are all of the type of Eq. (4.6), where $\mathcal{X}(x)$ is one of the eMPLs appearing in Eq. (3.19). Without loss of generality, we list here the integrals for $a_{i}=a_{1}$, while they will appear summed over all four roots $a_{i}$ in the primitive of $\mathcal{I}_{2}$.

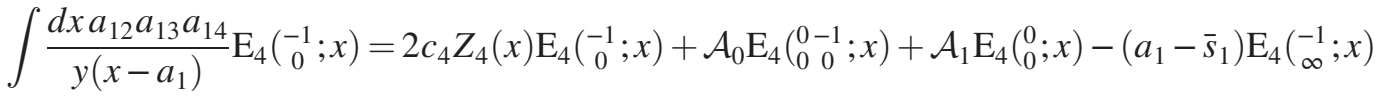

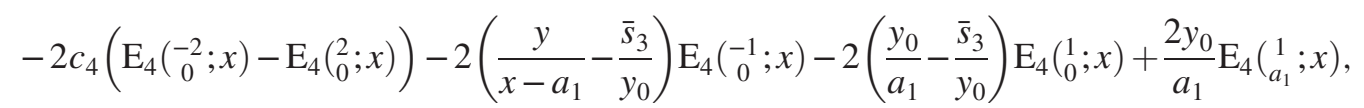

$$
\begin{aligned}
& \int \frac{d x a_{12} a_{13} a_{14}}{y\left(x-a_{1}\right)} \mathrm{E}_{4}\left(\begin{array}{c}
-1 \\
1
\end{array} ; x\right)=2 c_{4} Z_{4}(x) \mathrm{E}_{4}\left(\begin{array}{c}
-1 \\
1
\end{array} ; x\right)+\mathcal{A}_{0} \mathrm{E}_{4}\left(\begin{array}{c}
0-1 \\
0
\end{array} ; x\right)-2 c_{4} \mathrm{E}_{4}\left(\begin{array}{c}
-2 \\
1
\end{array} ; x\right)-2 \frac{y}{x-a_{1}} \mathrm{E}_{4}\left(\begin{array}{c}
-1 \\
1
\end{array} ; x\right) \\
& -2 \frac{y_{1}}{1-a_{1}}\left(\mathrm{E}_{4}\left(\begin{array}{c}
1 \\
a_{1}
\end{array} ; x\right)-\mathrm{E}_{4}\left(\begin{array}{l}
1 \\
1
\end{array} ; x\right)\right)
\end{aligned}
$$

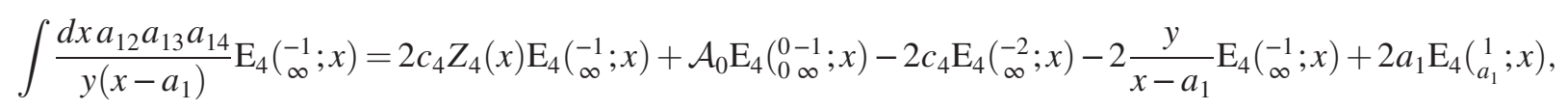

$$
\begin{aligned}
& \int \frac{d x a_{12} a_{13} a_{14}}{y\left(x-a_{1}\right)} \mathrm{E}_{4}\left(\begin{array}{l}
1 \\
0
\end{array} ; x\right)=2 c_{4}\left[Z_{4}(x)-Z_{4}(0)\right] \mathrm{E}_{4}\left(\begin{array}{l}
1 \\
0
\end{array} ; x\right)+\mathcal{A}_{0} \mathrm{E}_{4}\left(\begin{array}{ll}
0 & 1 \\
0 & 0
\end{array} x\right)-\mathcal{A}_{1} \mathrm{E}_{4}\left(\begin{array}{l}
0 \\
0
\end{array} ; x\right)-2 \frac{\bar{s}_{3}}{y_{0}} \mathrm{E}_{4}\left(\begin{array}{c}
-1 \\
0
\end{array} ; x\right)
\end{aligned}
$$

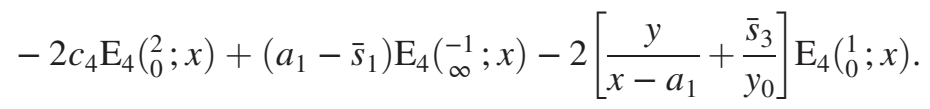

Here, we have defined the following abbreviations:

$$
\mathcal{A}_{0}=-\frac{3 a_{1}^{2}-2 a_{1} \bar{s}_{1}+\bar{s}_{2}}{3 c_{4}}-8 c_{4}^{2} \frac{\eta_{1}}{\omega_{1}} \quad \text { and } \quad \mathcal{A}_{1}=\frac{a_{1} \bar{s}_{1}-5 \bar{s}_{2}}{3 c_{4}}+\frac{8 c_{4} \eta_{1}}{\omega_{1}} .
$$

[1] A. B. Goncharov, Adv. Math. 114, 197 (1995).

[2] E. Remiddi and J. A. M. Vermaseren, Int. J. Mod. Phys. A 15, 725 (2000).

[3] A. B. Goncharov, arXiv:math/0103059v4.

[4] A. B. Goncharov, M. Spradlin, C. Vergu, and A. Volovich, Phys. Rev. Lett. 105, 151605 (2010).

[5] J. Ablinger, J. Blumlein, and C. Schneider, J. Math. Phys. 52, 102301 (2011).
[6] C. Duhr, H. Gangl, and J. R. Rhodes, J. High Energy Phys. 10 (2012) 075.

[7] C. Duhr, J. High Energy Phys. 08 (2012) 043.

[8] C. Duhr, in Proceedings, Theoretical Advanced Study Institute in Elementary Particle Physics: Journeys Through the Precision Frontier: Amplitudes for Colliders (TASI 2014): Boulder, Colorado (2015), p. 419, arXiv:1411.7538.

[9] A. Sabry, Nucl. Phys. 33, 401 (1962). 
[10] M. Czakon and A. Mitov, Nucl. Phys. B824, 111 (2010).

[11] A. von Manteuffel and L. Tancredi, J. High Energy Phys. 06 (2017) 127.

[12] M. Hidding and F. Moriello, arXiv:1712.04441.

[13] R. Bonciani, V. Del Duca, H. Frellesvig, J. M. Henn, F. Moriello, and V. A. Smirnov, J. High Energy Phys. 12 (2016) 096.

[14] M. Becchetti and R. Bonciani, J. High Energy Phys. 01 (2018) 048.

[15] S. Caron-Huot and K. J. Larsen, J. High Energy Phys. 10 (2012) 026.

[16] D. Nandan, M. F. Paulos, M. Spradlin, and A. Volovich, J. High Energy Phys. 05 (2013) 105.

[17] J. L. Bourjaily, A. J. McLeod, M. Spradlin, M. von Hippel, and M. Wilhelm, arXiv:1712.02785.

[18] D. J. Broadhurst, Z. Phys. C 47, 115 (1990).

[19] S. Bauberger, F. A. Berends, M. Bohm, and M. Buza, Nucl. Phys. B434, 383 (1995).

[20] S. Bauberger and M. Bohm, Nucl. Phys. B445, 25 (1995).

[21] S. Laporta and E. Remiddi, Nucl. Phys. B704, 349 (2005).

[22] B. A. Kniehl, A. V. Kotikov, A. Onishchenko, and O. Veretin, Nucl. Phys. B738, 306 (2006).

[23] S. Bloch and P. Vanhove, J. Number Theory 148, 328 (2015).

[24] L. Adams, C. Bogner, and S. Weinzierl, J. Math. Phys. 54, 052303 (2013).

[25] E. Remiddi and L. Tancredi, Nucl. Phys. B880, 343 (2014).

[26] L. Adams, C. Bogner, and S. Weinzierl, J. Math. Phys. 55, 102301 (2014).

[27] L. Adams, C. Bogner, and S. Weinzierl, J. Math. Phys. 56, 072303 (2015).

[28] L. Adams, C. Bogner, and S. Weinzierl, J. Math. Phys. 57, 032304 (2016).

[29] E. Remiddi and L. Tancredi, Nucl. Phys. B907, 400 (2016).

[30] L. Adams, C. Bogner, A. Schweitzer, and S. Weinzierl, J. Math. Phys. 57, 122302 (2016).

[31] E. Remiddi and L. Tancredi, Nucl. Phys. B925, 212 (2017).
[32] G. Passarino, Eur. Phys. J. C 77, 77 (2017).

[33] L.-B. Chen, J. Jiang, and C.-F. Qiao, arXiv:1712.03516.

[34] A. Beilinson and A. Levin, in Proceedings of Symposia in Pure Mathematics 55, Part II, edited by J.-P. S. U. Jannsen and S. L. Kleiman (American Mathematical Society, Providence, 1994), p. 123.

[35] A. Levin and G. Racinet, arXiv:math/0703237.

[36] F. Brown and A. Levin, arXiv:1110.6917.

[37] J. Broedel, C. R. Mafra, N. Matthes, and O. Schlotterer, J. High Energy Phys. 07 (2015) 112.

[38] J. Broedel, N. Matthes, and O. Schlotterer, J. Phys. A 49, 155203 (2016).

[39] J. Broedel, N. Matthes, G. Richter, and O. Schlotterer, arXiv:1704.03449.

[40] J. Broedel, C. Duhr, F. Dulat, and L. Tancredi, arXiv: 1712.07095 .

[41] O. V. Tarasov, Phys. Rev. D 54, 6479 (1996).

[42] R. N. Lee, Nucl. Phys. B830, 474 (2010).

[43] N. Matthes, Ph.D. thesis, Universität Hamburg, 2016.

[44] We have also performed the computations in the differentmass case from Feynman parameters. While both approaches give the same results numerically, the results obtained for $S_{211}$ with different masses lead to a rather complicated and lengthy analytic expression.

[45] S. Borowka, G. Heinrich, S. P. Jones, M. Kerner, J. Schlenk, and T. Zirke, Comput. Phys. Commun. 196, 470 (2015).

[46] S. Borowka, G. Heinrich, S. Jahn, S. P. Jones, M. Kerner, J. Schlenk, and T. Zirke, Comput. Phys. Commun. 222, 313 (2018).

[47] A. V. Kotikov, Phys. Lett. B 254, 158 (1991).

[48] Z. Bern, L. J. Dixon, and D. A. Kosower, Nucl. Phys. B412, 751 (1994).

[49] E. Remiddi, Nuovo Cimento A 110, 1435 (1997).

[50] T. Gehrmann and E. Remiddi, Nucl. Phys. B580, 485 (2000). 